\title{
REAL FUNCTION ALGEBRAS
}


PURE AND APPLIED MATHEMATICS

A Program of Monographs, Textbooks, and Lecture Notes

\title{
EXECUTIVE EDITORS
}

\author{
Earl J. Taft \\ Rutgers University \\ New Brunswick, New Jersey
}

\author{
Zuhair Nashed \\ University of Delaware \\ Newark, Delaware
}

\section{CHAIRMEN OF THE EDITORIAL BOARD}

S. Kobayashi

University of California, Berkeley

Berkeley, California
Edwin Hewitt

University of Washington

Seattle, Washington

\section{EDITORIAL BOARD}

M. S. Baouendi

University of California, San Diego

Jack K. Hale

Georgia Institute of Technology

Marvin Marcus

University of California,

Santa Barbara

W. S. Massey

Yale University

Leopoldo Nachbin Centro Brasileiro de

Pesquisas Fisicas

Anil Nerode

Cornell University
Donald Passman

University of Wisconsin-Madison

Fred S. Roberts

Rutgers University

Gian-Carlo Rota

Massachusetts Institute of

Technology

David L. Russell

Virginia Polytechnic Institute

and State University

Jane Cronin Scanlon

Rutgers University

Walter Schempp

Universität Siegen

Mark Tepty

University of Wisconsin-Milwaukee 


\section{MONOGRAPHS AND TEXTBOOKS IN PURE AND APPLIED MATHEMATICS}

1. K. Yano, Integral Formulas in Riemannian Geometry (1970)

2. S. Kobayashi, Hyperbolic Manifolds and Holomorphic Mappings (1970)

3. V. S. Vladimirov, Equations of Mathematical Physics (A. Jeffrey, ed.; A. Littlewood, trans.) (1970)

4. B. N. Pshenichnyi, Necessary Conditions for an Extremum (L. Neustadt, translation ed.; K. Makowski, trans.) (1971)

5. L. Narici et al., Functional Analysis and Valuation Theory (1971)

6. S. S. Passman, Infinite Group Rings (1971)

7. L. Dornhoff, Group Representation Theory. Part A: Ordinary Representation Theory. Part B: Modular Representation Theory (1971, 1972)

8. W. Boothby and G. L. Weiss, eds., Symmetric Spaces (1972)

9. Y. Matsushima, Differentiable Manifolds (E. T. Kobayashi, trans.) (1972)

10. L. E. Ward, Jr., Topology (1972)

11. A. Babakhanian, Cohomological Methods in Group Theory (1972)

12. R. Gilmer, Multiplicative Ideal Theory (1972)

13. J. Yeh, Stochastic Processes and the Wiener Integral (1973)

14. J. Barros-Neto, Introduction to the Theory of Distributions (1973)

15. R. Larsen, Functional Analysis (1973)

16. K. Yano and S. Ishihara, Tangent and Cotangent Bundles (1973)

17. C. Procesi, Rings with Polynomial Identities (1973)

18. R. Hermann, Geometry, Physics, and Systems (1973)

19. N. R. Wallach, Harmonic Analysis on Homogeneous Spaces (1973)

20. J. Dieudonné, Introduction to the Theory of Formal Groups (1973)

21. I. Vaisman, Cohomology and Differential Forms (1973)

22. B.-Y. Chen, Geometry of Submanifolds (1973)

23. M. Marcus, Finite Dimensional Multilinear Algebra (in two parts) $(1973,1975)$

24. R. Larsen, Banach Algebras (1973)

25. R. O. Kujala and A. L. Vitter, eds., Value Distribution Theory: Part A; Part B: Deficit and Bezout Estimates by Wilhelm Stoll (1973)

26. K. B. Stolarsky, Algebraic Numbers and Diophantine Approximation (1974)

27. A. R. Magid, The Separable Galois Theory of Commutative Rings (1974)

28. B. R. McDonald, Finite Rings with Identity (1974)

29. J. Satake, Linear Algebra (S. Koh et al., trans.) (1975)

30. J. S. Golan, Localization of Noncommutative Rings (1975)

31. G. Klambauer, Mathematical Analysis (1975)

32. M. K. Agoston, Algebraic Topology (1976)

33. K. R. Goodearl, Ring Theory (1976)

34. L. E. Mansfield, Linear Algebra with Geometric Applications (1976)

35. N. J. Pullman, Matrix Theory and its Applications (1976)

36. B. R. McDonald, Geometric Algebra Over Local Rings (1976)

37. C. W. Groetsch, Generalized Inverses of Linear Operators (1977)

38. J. E. Kuczkowski and J. L. Gersting, Abstract Algebra (1977)

39. C. O. Christenson and W. L. Voxman, Aspects of Topology (1977)

40. M. Nagata, Field Theory (1977)

41. R. L. Long, Algebraic Number Theory (1977)

42. W. F. Pfeffer, Integrals and Measures (1977)

43. R. L. Wheeden and A. Zygmund, Measure and Integral (1977)

44. J. H. Curtiss, Introduction to Functions of a Complex Variable (1978)

45. K. Hrbacek and T. Jech, Introduction to Set Theory (1978)

46. W. S. Massey, Homology and Cohomology Theory (1978)

47. M. Marcus, Introduction to Modern Algebra (1978)

48. E. C. Young, Vector and Tensor Analysis (1978)

49. S. B. Nadler, Jr., Hyperspaces of Sets (1978)

50. S. K. Segal, Topics in Group Kings (1978) 
51. A. C. M. van Rooij, Non-Archimedean Functional Analysis (1978)

52. L. Corwin and R. Szczarba, Calculus in Vector Spaces (1979)

53. C. Sadosky, Interpolation of Operators and Singular Integrals (1979)

54. J. Cronin, Differential Equations (1980)

55. C. W. Groetsch, Elements of Applicable Functional Analysis (1980)

56. 1. Vaisman, Foundations of Three-Dimensional Euclidean Geometry (1980)

57. H. I. Freedan, Deterministic Mathematical Models in Population Ecology (1980)

58. S. B. Chae, Lebesgue Integration (1980)

59. C. S. Rees et al., Theory and Applications of Fourier Analysis (1981)

60. L. Nachbin, Introduction to Functional Analysis (R. M. Aron, translator) (1981)

61. G. Orzech and M. Orzech, Plane Algebraic Curves (1981)

62. R. Johnsonbaugh and W. E. Pfaffenberger, Foundations of Mathematical Analysis (1981)

63. W. L. Voxman and R. H. Goetschel, Advanced Calculus (1981)

64. L. J. Corwin and R. H. Szcarba, Multivariable Calculus (1982)

65. V. I. Istrățescu, Introduction to Linear Operator Theory (1981)

66. R. D. Järvinen, Finite and Infinite Dimensional Linear Spaces (1981)

67. J. K. Beem and P. E. Ehrlich, Global Lorentzian Geometry (1981)

68. D. L. Armacost, The Structure of Locally Compact Abelian Groups (1981)

69. J. W. Brewer and M. K. Smith, eds., Emily Noether: A Tribute (1981)

70. K. H. Kim, Boolean Matrix Theory and Applications (1982)

71. T. W. Wieting, The Mathematical Theory of Chromatic Plane Ornaments (1982)

72. D. B. Gauld, Differential Topology (1982)

73. R. L. Faber, Foundations of Euclidean and Non-Euclidean Geometry (1983)

74. M. Carmeli, Statistical Theory and Random Matrices (1983)

75. J. H. Carruth et al., The Theory of Topological Semigroups (1983)

76. R. L. Faber, Differential Geometry and Relativity Theory (1983)

77. S. Barnett, Polynomials and Linear Control Systems (1983)

78. G. Karpilovsky, Commutative Group Algebras (1983)

79. F. Van Oystaeyen and A. Verschoren, Relative Invariants of Rings (1983)

80. 1. Vaisman, A First Course in Differential Geometry (1984)

81. G. W. Swan, Applications of Optimal Control Theory in Biomedicine (1984)

82. T. Petrie and J. D. Randall, Transformation Groups on Manifolds (1984)

83. K. Goebel and S. Reich, Uniform Convexity, Hyperbolic Geometry, and Nonexpansive Mappings (1984)

84. T. Albu and C. Năstăsescu, Relative Finiteness in Module Theory (1984)

85. K. Hrbacek and T. Jech, Introduction to Set Theory: Second Edition (1984)

86. F. Van Oystaeyen and A. Verschoren, Relative Invariants of Rings (1984)

87. B. R. McDonald, Linear Algebra Over Commutative Rings (1984)

88. M. Namba, Geometry of Projective Algebraic Curves (1984)

89. G. F. Webb, Theory of Nonlinear Age-Dependent Population Dynamics (1985)

90. M. R. Bremner et al., Tables of Dominant Weight Multiplicities for Representations of Simple Lie Algebras (1985)

91. A. E. Fekete, Real Linear Algebra (1985)

92. S. B. Chae, Holomorphy and Calculus in Normed Spaces (1985)

93. A. J. Jerri, Introduction to Integral Equations with Applications (1985)

94. G. Karpilovsky, Projective Representations of Finite Groups (1985)

95. L. Narici and E. Beckenstein, Topological Vector Spaces (1985)

96. J. Weeks, The Shape of Space (1985)

97. P. R. Gribik and K. O. Kortanek, Extremal Methods of Operations Research (1985)

98. J.-A. Chao and W. A. Woyczynski, eds., Probability Theory and Harmonic Analysis (1986)

99. G. D. Crown et al., Abstract Algebra (1986)

100. J. H. Carruth et al., The Theory of Topological Semigroups, Volume 2 (1986)

101. R. S. Doran and V. A. Belfi, Characterizations of C*-Algebras (1986)

102. M. W. Jeter, Mathematical Programming (1986)

103. M. Altman, A Unified Theory of Nonlinear Operator and Evolution Equations with Applications (1986)

104. A. Verschoren, Relative Invariants of Sheaves (1987) 
105. R. A. Usmani, Applied Linear Algebra (1987)

106. P. Blass and J. Lang, Zariski Surfaces and Differential Equations in Characteristic $p$ $>0$ (1987)

107. J. A. Reneke et al., Structured Hereditary Systems (1987)

108. H. Busemann and B. B. Phadke, Spaces with Distinguished Geodesics (1987)

109. R. Harte, Invertibility and Singularity for Bounded Linear Operators (1988)

110. G. S. Ladde et al., Oscillation Theory of Differential Equations with Deviating Arguments (1987)

111. L. Dudkin et al., Iterative Aggregation Theory (1987)

112. T. Okubo, Differential Geometry (1987)

113. D. L. Stancl and M. L. Stancl, Real Analysis with Point-Set Topology (1987)

114. T. C. Gard, Introduction to Stochastic Differential Equations (1988)

115. S. S. Abhyankar, Enumerative Combinatorics of Young Tableaux (1988)

116. H. Strade and R. Farnsteiner, Modular Lie Algebras and Their Representations (1988)

117. J. A. Huckaba, Commutative Rings with Zero Divisors (1988)

118. W. D. Wallis, Combinatorial Designs (1988)

119. W. Więslaw, Topological Fields (1988)

120. G. Karpilovsky, Field Theory (1988)

121. S. Caenepeel and F. Van Oystaeyen, Brauer Groups and the Cohomology of Graded Rings (1989)

122. W. Kozlowski, Modular Function Spaces (1988)

123. E. Lowen-Colebunders, Function Classes of Cauchy Continuous Maps (1989)

124. M. Pavel, Fundamentals of Pattern Recognition (1989)

125. V. Lakshmikantham et al., Stability Analysis of Nonlinear Systems (1989)

126. R. Sivaramakrishnan. The Classical Theory of Arithmetic Functions (1989)

127. N. A. Watson, Parabolic Equations on an Infinite Strip (1989)

128. K. J. Hastings, Introduction to the Mathematics of Operations Research (1989)

129. B. Fine, Algebraic Theory of the Bianchi Groups (1989)

130. D. N. Dikranjan et al., Topological Groups (1989)

131. J. C. Morgan II, Point Set Theory (1990)

132. P. Biler and A. Witkowski, Problems in Mathematical Analysis (1990)

133. H. J. Sussmann, Nonlinear Controllability and Optimal Control (1990)

134. J.-P. Florens et al., Elements of Bayesian Statistics (1990)

135. N. Shell, Topological Fields and Near Valuations (1990)

136. B. F. Doolin and C. F. Martin, Introduction to Differential Geometry for Engineers (1990)

137. S. S. Holland, Jr., Applied Analysis by the Hilbert Space Method (1990)

138. J. Okniniski, Semigroup Algebras (1990)

139. K. Zhu, Operator Theory in Function Spaces (1990)

140. G. B. Price, An introduction to Multicomplex Spaces and Functions (1991)

141. R. B. Darst, Introduction to Linear Programming (1991)

142. P. L. Sachdev, Nonlinear Ordinary Differential Equations and Their Applications (1991)

143. T. Husain, Orthogonal Schauder Bases (1991)

144. J. Foran, Fundamentals of Real Analysis (1991)

145. W. C. Brown, Matrices and Vector Spaces (1991)

146. M. M. Rao and Z. D. Ren, Theory of Orlicz Spaces (1991)

147. J. S. Golan and T. Head, Modules and the Structures of Rings (1991)

148. C. Small, Arithmetic of Finite Fields (1991)

149. K. Yang, Complex Algebraic Geometry (1991)

150. D. G. Hoffman et al., Coding Theory (1991)

151. M. O. González, Classical Complex Analysis (1992)

152. M. O. González, Complex Analysis (1992)

153. L. W. Baggett, Functional Analysis (1992)

154. M. Sniedovich, Dynamic Programming (1992)

155. R. P. Agarwal, Difference Equations and Inequalities (1992)

156. C. Brezinski, Biorthogonality and Its Applications to Numerical Analysis (1992)

157. C. Swartz, An Introduction to Functional Analysis (1992)

158. S. B. Nadler, Jr., Continuum Theory (1992) 
159. M. A. Al-Gwaiz, Theory of Distributions (1992)

160. E. Perry, Geometry: Axiomatic Developments with Problem Solving (1992)

161. E. Castillo and M. R. Ruiz-Cobo, Functional Equations and Modelling in Science and Engineering (1992)

162. A. J. Jerri, Integral and Discrete Transforms with Applications and Error Analysis (1992)

163. A. Charlier et al., Tensors and the Clifford Algebra (1992)

164. P. Biler and T. Nadzieja, Problems and Examples in Differential Equations (1992)

165. E. Hansen, Global Optimization Using Interval Analysis (1992)

166. S. Guerre-Delabrière, Classical Sequences in Banach Spaces (1992)

167. Y. C. Wong, Introductory Theory of Topological Vector Spaces (1992)

168. S. H. Kulkarni and B. V. Limaye, Real Function Algebras (1992)

Additional Volumes in Preparation 


\section{REAL FUNCTION ALGEBRAS}

\section{S. H. Kulkarni}

Indian Institute of Technology

Madras, India

\section{B.V. Limaye}

Indian Institute of Technology

Bombay, India

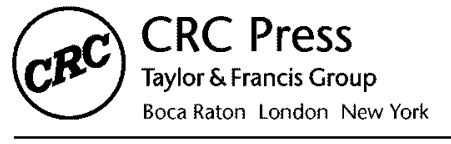

CRC Press is an imprint of the

Taylor \& Francis Group, an informa business 
CRC Press

Taylor \& Francis Group

6000 Broken Sound Parkway NW, Suite 300

Boca Raton, FL 33487-2742

First issued in paperback 2019

(C) 1992 by Taylor \& Francis Group, LLC

CRC Press is an imprint of Taylor \& Francis Group, an Informa business

No claim to original U.S. Government works

ISBN-13: 978-0-8247-8653-3 (hbk)

ISBN-13: 978-0-367-40271-6 (pbk)

This book contains information obtained from authentic and highly regarded sources. Reasonable efforts have been made to publish reliable data and information, but the author and publisher cannot assume responsibility for the validity of all materials or the consequences of their use. The authors and publishers have attempted to trace the copyright holders of all material reproduced in this publication and apologize to copyright holders if permission to publish in this form has not been obtained. If any copyright material has not been acknowledged please write and let us know so we may rectify in any future reprint.

Except as permitted under U.S. Copyright Law, no part of this book may be reprinted, reproduced, transmitted, or utilized in any form by any electronic, mechanical, or other means, now known or hereafter invented, including photocopying, microfilming, and recording, or in any information storage or retrieval system, without written permission from the publishers.

For permission to photocopy or use material electronically from this work, please access www.copyright.com (http://www.copyright.com/) or contact the Copyright Clearance Center, Inc. (CCC), 222 Rosewood Drive, Danvers, MA 01923, 978-750-8400. CCC is a not-for-profit organization that provides licenses and registration for a variety of users. For organizations that have been granted a photocopy license by the $\mathrm{CCC}$, a separate system of payment has been arranged.

Trademark Notice: Product or corporate names may be trademarks or registered trademarks, and are used only for identification and explanation without intent to infringe.

\section{Library of Congress Cataloging-in-Publication Data}

\section{Kulkarni, S. H.}

Real function algebras / S. H. Kulkarni, B. V. Limaye.

p. cm. - (Monographs and textbooks in pure and applied mathematics : 168)

Includes bibliographical references and index.

ISBN 0-8247-8653-X

1. Function algebras. I. Limaye, Balmohan Vishnu. II. Title. III. Series.

QA326.K85 1992

512'.55-dc20

Visit the Taylor \& Francis Web site at

http://www.taylorandfrancis.com

and the CRC Press Web site at

http://www.crepress.com 


\section{Preface}

The beautiful theory of complex function algebras has been developed extensively over the past thirty years or so. These are the uniformly closed complex subalgebras of $C(X)$ separating the points of $X$ and containing the constants, where $X$ is a compact Hausdorff space. The enrichment of this theory has come largely from the theory of complex analytic functions. This area now occupies a proud place in the general theory of complex Banach algebras. On the other hand, real function algebras (and, for that matter, real Banach algebras in general) have received relatively scant attention. These are the uniformly closed real subalgebras of $C(X, \tau)$ separating the points of $X$ and containing the constants, where $\tau$ is a continuous involution on $X$ and $C(X, \tau)=\{f \in C(X): f \circ \tau=\bar{f}\}$.

Our aim in writing this book is to give an account of the present theory of real function algebras. This theory has been developed essentially during the last ten years in analogy with the theory of complex function algebras. There are two distinct approaches to this development. One is the so-called complexification technique: If $A$ is a real function algebra on $(X, \tau)$, then its complexification $B=\{f+i g: f, g$ in $A\}$ is a complex function algebra on $X$, and results for $A$ can often be deduced from the corresponding results for $B$. The other approach is to prove results for $A$ intrinsically, without resorting to its complexification. Although the intrinsic approach is, in general, more difficult than the former, we have preferred it on aesthetic grounds. Also, since every complex function 
algebra can be viewed as a real function algebra, this approach at once generalizes the theory of complex function algebras. Only when the intrinsic approach seemed too difficult is recourse made to the complexification technique. A reader of this book is not required to be already conversant with the theory of complex function algebras, except in isolated instances. In fact, even the prerequisites from the theory of real and complex Banach algebras are developed in the first three sections of Chapter 1. The book is addressed to those readers who have a brief introduction to real and complex variables and to functional analysis. It may enable them to employ their knowledge to obtain further results concerning continuous, analytic, and harmonic functions.

At the beginning of each chapter, we have summarized its contents. The dependence of various sections of the book is indicated in the following diagram.

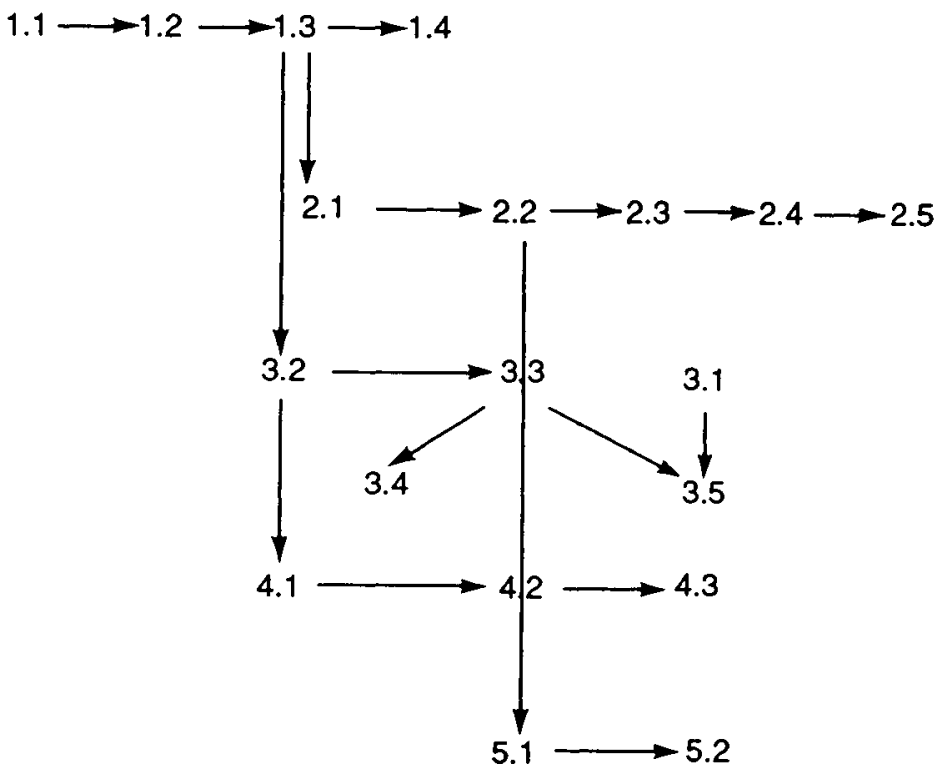

The present extent of the theory of real function algebras (and hence the scope of this book) is rather limited. Real analogs of several interesting complex results remain to be explored. Even among the known real results, we discuss only those that are in our view basic. However, we have tried to be exhaustive in giving references to related results.

It seems worthwhile to point out some results in this book that are of 
general mathematical interest: topological conditions for the commutativity of a real or complex Banach algebra (Theorem 1.1.30), Ransford's short elementary proof of the Bishop-Stone-Weierstrass theorem (Theorem 2.1.2), a result implying the analyticity or the antianalyticity of a function $f$ from the harmonicity of $\operatorname{Re} f, \operatorname{Re} f^{2}, \operatorname{Re} f^{3}$, and $\operatorname{Re} f^{4}$ (Theorem 3.1.3), and a result regarding the positivity of a real linear functional on a real subspace of $C(X)$ (Lemma 3.2.4).

We thank the Directors of the Indian Institutes of Technology at Bombay and Madras for making it possible for us to work together under the Inter I.I.T. Faculty Exchange Programme. We acknowledge the support of the Curriculum Development Cell at the I.I.T. Bombay and the Centre for Continuing Education at the I.I.T. Madras in preparing the manuscript, and of Marcel Dekker, Inc., New York, in publishing this book. We are indebted to R. C. Burckel and K. Jarosz for their encouragement. We also thank M. Parameswaran and M. M. Thomas for typing the manuscript, and Sushama Aggrawal, S. Arundhathi, K. C. Sivakumar, M. H. Vasavada, and P. Veeramani for reading it critically. Finally, we are grateful to Chaya Kulkarni and Nirmala Limaye, who kept our morale high during the preparation of this work.

S. H. Kulkarni

B. V. Limaye 


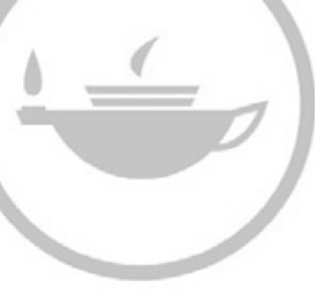

$$
\begin{aligned}
& \text { Taylor \& Francis } \\
& \text { Taylor \& Francis Group }
\end{aligned}
$$

http://taylorandfrancis.com 


\section{Contents}

$\begin{array}{lll}\text { PREFACE } & \text { iii }\end{array}$

1. INTRODUCTION 1

1.1 Elements of Real Banach Algebras 1

1.2 Commutative Real Banach Algebras 19

1.3 Real Function Algebras 27

1.4 Deformations of a Real Function Algebra 39

2. WHEN DOES A REAL FUNCTION ALGEBRA EQUAL $C(X, \tau)$ ? 46

2.1 Analog of Bishop-Machado Theorem 47

2.2 Antisymmetric Sets and Peak Sets 60

2.3 Hoffman-Wermer Theorem and Its Analog 69

2.4 Analog of Wermer's Theorem 74

2.5 Functions that Operate in $\operatorname{Re} A \quad 80$

3. GLEASON PARTS OF A REAL FUNCTION ALGEBRA $\quad 89$

3.1 Analytic and Harmonic Structure 90

3.2 Characterizations of Gleason Parts 95 
3.3 Parts of the Complexification

3.4 Topological Characterization of Gleason Parts

3.5 Parts, Analyticity, and Harmonicity

4. BOUNDARIES FOR A REAL FUNCTION ALGEBRA

4.1 Choquet Sets for Subspaces of $C(X, \tau)$

4.2 Boundaries for Subspaces of $C(X, \tau)$

4.3 Choquet Sets and Boundaries for Complex Subspaces of $C(X)$

5. ISOMETRIES OF REAL FUNCTION ALGEBRAS 164

5.1 Unit-Preserving Isometries 165

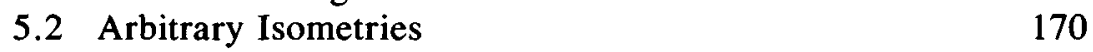

$\begin{array}{ll}\text { SYMBOLS } & 175\end{array}$

$\begin{array}{ll}\text { REFERENCES } & 177\end{array}$

$\begin{array}{ll}\text { INDEX } & 183\end{array}$ 


\section{1}

\section{Introduction}

In this monograph we are concerned with real function algebras, which are, in particular, Banach algebras. For this reason we begin by developing the necessary theory of real Banach algebras. Another reason for developing this general theory is that in the study of real function algebras, we have to deal with some Banach algebras that are not real function algebras.

The first section contains a definition and some examples of Banach algebras, properties of the spectrum of an element, the spectral radius formula, functional calculus, and some conditions for the commutativity of a Banach algebra. The second section deals with the representation of a commutative real Banach algebra with a unit element as an algebra of continuous functions on a compact Hausdorff space. We define a real function algebra in the third section and give several examples. Some properties of real function algebras are also discussed. These three sections are an essential prerequisite for the remainder of the book. The final section introduces the relatively recently developed topic of perturbation of Banach algebras. It is not used later in this book.

\subsection{ELEMENTS OF REAL BANACH ALGEBRAS}

Definition 1.1.1 An algebra $A$ over a field $F$ is a ring which is also a vector space over $F$ such that $(\alpha a)(b)=\alpha(a b)=(a)(\alpha b)$ for all $a, b$ in $A$ and $\alpha$ in F. $A$ is said to be commutative if $a b=b a$ for $a$ and $b$ in $A$. 
An algebra $A$ is called a real algebra if $\beta=\mathbb{R}$, the field of real numbers, and a complex algebra if $F=\mathbb{C}$, the field of complex numbers; such an algebra is said to be normed by $\|\cdot\|$ if $(A,\|\cdot\|)$ is a normed linear space such that

$$
\|a b\| \leq\|a\|\|b\|
$$

for all $a, b$ in $A$; if $A$ has a unit element 1 (i.e., $1 a=a=a 1$ for all $a$ in $A$ ), it is unique and $\|1\| \geq 1$. We can, in fact, assume that $\|1\|=1$ since $\|a\|_{1}=\sup \{\|a b\| /\|b\|: 0 \neq b$ in $A\}$ is an equivalent norm on $A$ for which $\|1\|_{1}=1$.

If every Cauchy sequence in a normed algebra $A$ converges in $A$, then $A$ is called a Banach algebra. Note that every complex Banach algebra $A$ can be considered as a real Banach algebra $A_{R}$. Later we shall consider a natural way of constructing a complex Banach algebra from a real Banach algebra.

Example 1.1.2 Let $X$ be a compact Hausdorff topological space. By $C(X)$ we denote the set of all complex-valued continuous functions on $X$. Under pointwise operations, $C(X)$ is a complex commutative algebra and has the constant function 1 as the unit element. For $f \in C(X)$, we define

$$
\|f\|:=\sup \{|f(x)|: x \in X\} .
$$

It is easy to see that $C(X)$ is a complex Banach algebra.

As examples of closed real subalgebras that are not complex subalgebras, we may consider the following.

(a) Let $Y$ be a closed subset of $X$. Consider

$$
C_{Y}:=\{f \in C(X): f(Y) \subset\{\} .
$$

If $Y$ is the empty set, $C_{Y}=C(X)$. For every nonempty set $Y, C_{Y}$ is a real commutative Banach algebra. When $Y=X$, we get the algebra of all continuous real-valued functions on $X$. We denote this algebra by $C_{\mathbb{R}}(X)$. (b) Let $\tau: X \rightarrow X$ be a homeomorphism such that $\tau^{2}=\tau \circ \tau$ is the identity map on $X$. Let

$$
C(X, \tau):=\{f \in C(X): f(\tau(x))=\tilde{f}(x) \text { for all } x \in X\} .
$$

Then $C(X, \tau)$ is a real Banach algebra. It is not a complex algebra. Throughout the book we shall have frequent occasions to deal with this algebra.

Note that if $\tau$ is the identity map on $X$, then $C(X, \tau)=C_{\text {品 }}(X)$. In fact, every example of type (a) can be transformed into one of type (b) by constructing a new space from one copy of $Y$ and two copies of $X \backslash Y$. 
However, not all examples of type (b) can be put in the form of type (a). [See Exercises 8A and 8B of Goodearl (1982) for details.]

Example 1.1.3 Let $X \neq\{0\}$ be a (real or complex) normed linear space, and let $B L(X)$ denote the set of all bounded linear operators on $X$. Under the usual operations $B L(X)$ is a noncommutative algebra. It has a unit element, namely, the identity operator, which we denote by $I$. Further, for $T \in B L(X)$, we define the operator norm by

$$
\|T\|:=\sup \{\|T(x)\|: x \in X, \quad\|x\| \leq 1\} .
$$

Then $(B L(X),\|\cdot\|)$ is a normed algebra. It is a Banach algebra if and only if $X$ is a Banach space.

In particular, if $X=\mathbb{R}^{n}$ (respectively, $\left.\mathbb{C}^{n}\right)$, then $B L(X)$ can be identified, in the usual way, with the algebra of all $n \times n$ matrices with real (respectively, complex) entries.

Example 1.1.4 Let $I=(1,0,0,0), \mathbf{i}=(0,1,0,0), \mathbf{j}=(0,0,1,0)$, and $k=(0,0,0,1)$ denote the basis vectors of $R^{4}$. We define

$$
\begin{gathered}
\mathbf{l}^{2}=\mathbf{l}, \quad \mathbf{l} \mathbf{i}=\mathbf{i}=\mathbf{i l}, \quad \mathbf{l} \mathbf{j}=\mathbf{j}=\mathbf{j} \mathbf{l}, \quad \mathbf{l} \mathbf{k}=\mathbf{k}=\mathbf{k} \mathbf{l}, \\
\mathbf{i}^{2}=\mathbf{j}^{2}=\mathbf{k}^{2}=-\mathbf{l}, \quad \mathbf{i j}=-\mathbf{j} \mathbf{i}=\mathbf{k}, \quad \mathbf{j k}=-\mathbf{k} \mathbf{j}=\mathbf{i}, \\
\mathbf{k i}=-\mathbf{i k}=\mathbf{j},
\end{gathered}
$$

and extend this product to $\Omega^{4}$ by linearity. Then we obtain a real noncommutative algebra, known as the real quaternion algebra 느. For $a=a_{0} \mathbf{l}+$ $a_{1} \mathbf{i}+a_{2} \mathbf{j}+a_{3} \mathbf{k}$ in $M$, we define $\|a\|:=\left(a_{0}^{2}+a_{1}^{2}+a_{2}^{2}+a_{3}^{2}\right)^{1 / 2}$. It is routine to check that $(\mathbb{N},\|\cdot\|)$ is a real Banach algebra with the unit element 1. In fact, for $a, b$ in $几$, $\|a b\|=\|a\|\|b\|$.

Example 1.1.5 Let $W$ be the set of all complex-valued functions on $[0,2 \pi]$ whose Fourier series are absolutely convergent, that is, functions of the form

$$
f(t)=\sum_{n=-\infty}^{\infty} c_{n} \exp (\text { int }), \quad t \in[0,2 \pi],
$$

where $c_{n}$ is a complex number for each $n$ and $\Sigma_{n=-\infty}^{\infty}\left|c_{n}\right|$ is finite. For such a function $f$, we define

$$
\|f\|=\sum_{n=-\infty}^{\infty}\left|c_{n}\right|
$$

Under pointwise operations, $W$ is a complex Banach algebra. This is 
known as the Wiener algebra, partly because of the following theorem of Wiener: If $f \in W$ and $f(t) \neq 0$ for all $t \in[0,2 \pi]$, then $1 / f \in W$. Gelfand gave an elegant proof of this result in his fundamental paper of 1941.

The complex Banach algebras have received far greater attention than the real Banach algebras. The main reason seems to be the possibility of using the power of highly developed analytic function theory via the Gelfand transformation. Scalar multiplication in a complex algebra $A$ is a map from $\mathbb{C} \times A$ to $A$. By considering the restriction of this map to $\mathrm{R} \times$ $A, A$ can be viewed as a real algebra. Hence every complex Banach algebra is also a real Banach algebra. Thus it is natural to ask what can be said about this larger class. However, in the classical literature one can find only incidental remarks on the real scalar case. There are a few exceptions to this pattern: the articles of Arens and Kaplansky (1948), Kadison (1951), and Kaplansky (1949). In Rickart's treatise (1960), a large part of the theory is developed for real and complex scalars simultaneously. The books of Bonsall and Duncan (1973) and Zelazko (1973) contain a few sections about real Banach algebras. About a third of the monograph of Goodearl (1982) is devoted to a particular type of real Banach algebras: real $C^{*}$-algebras. The first systematic exposition of real Banach algebras was given by Ingelstam (1964). Ingelstam has dealt with many problems in real Banach algebras $(1962,1963,1966,1967,1969)$. In the 1970s, further attention to real Banach algebras was paid in Alling (1970), Alling and Greenleaf (1971), Alling and Campbell (1972), Limaye (1975), Limaye and Simha (1975), Oliver (1970), Ono (1970), and Palmer (1972).

We now proceed to develop some parts of the theory of real Banach algebras by first describing a procedure for studying a real Banach algebra via its complexification.

Definition 1.1.6 Given a real algebra $A$, the complexification $B$ of $A$ is the set $A \times A$ with the operations of addition, multiplication, and scalar multiplication defined by

$$
\begin{aligned}
(a, b)+(c, d) & :=(a+c, b+d), \\
(\alpha+i \beta)(a, b) & :=(\alpha a-\beta b, \alpha b+\beta a), \\
(a, b)(c, d) & :=(a c-b d, a d+b c),
\end{aligned}
$$

for all $a, b, c, d$ in $A$ and $\alpha, \beta$ in $\Omega$.

It is routine to check that $B$ is a complex algebra. If $(A,\|\cdot\|)$ is a real normed algebra, it is possible to construct a norm $p$ on $B$ satisfying the 
following properties:

(i) $(B, p)$ is a complex normed algebra and $(B, p)$ is Banach whenever $(A,\|\cdot\|)$ is Banach.

(ii) $\max (\|a\|,\|b\|) \leq p((a, b)) \leq 2 \max (\|a\|,\|b\|)$ for $a, b$ in $A$.

(iii) $p((a, 0))=\|a\|$ for $a$ in $A$.

Since we shall not have any occasion to use this normed complexification of an arbitrary real normed algebra in this book, we do not bother to prove the foregoing assertion. Interested readers can find a proof in Bonsall and Duncan (1973). A usual method of studying a real Banach algebra $A$ is to consider the complexification $B$ of $A$ and then to apply known techniques of complex Banach algebras to $B$ in order to deduce results for the original algebra $A$. We shall refer to this method as the complexification technique. Arens and Kaplansky (1948), Rickart (1960), Bonsall and Duncan (1973), Alling (1970), and Ingelstam (1964) all use this technique primarily.

By an intrinsic method of studying real Banach algebras, we mean a method that does not resort to the complexification technique. It has a pedagogic advantage in the sense that one never goes outside the structure of a real Banach algebra and works only within it; however, there is no bar to using complex numbers and functions while employing the intrinsic method.

The concept of the spectrum of an element is basic in the theory of Banach algebras.

Definition 1.1.7 Let $A$ be a real or complex algebra with unit 1 and $a \in$ $A$. An element $b$ (respectively, $c$ ) is called a left (respectively, right) inverse of $a$ if $b a=1$ (respectively, $a c=1$ ). An inverse of $a$ is both a left and a right inverse of $a$. If $a$ has an inverse, $a$ is called invertible or regular; otherwise, it is called singular.

Definition 1.1.8 Let $A$ be a complex algebra with unit 1 and $a \in A$. Then the spectrum of $a$ in $A$ is the subset $\operatorname{Sp}(a, A)$ of $\mathbb{C}$ defined as follows:

$$
\operatorname{Sp}(a, A):=\{\lambda \in \mathbb{C}: a-\lambda \text { is singular in } A\} .
$$

Remark 1.1.9 Note that we have used the same symbol $\lambda$ for the scalar $\lambda$ and the element $\lambda \cdot 1$ of $A$. We shall continue this practice as the meaning is clear from the context. Further, we shall denote $\operatorname{Sp}(a, A)$ simply by $\operatorname{Sp}(a)$, when no emphasis on the algebra $A$ is required. This happens, for example, when we consider the spectra of various elements in the same algebra. 
It is natural to think that for a real algebra $A$ with unit 1 , the spectrum of an element $a$ in $A$ should be defined as the set of all real $\lambda$ for which $a-\lambda$ is singular in $A$. But if we accept this definition, the spectra of many elements may turn out to be the empty set. For example, if $A$ is the algebra of all $2 \times 2$ real matrices, and if

$$
a=\left[\begin{array}{rr}
0 & 1 \\
-1 & 0
\end{array}\right]
$$

then

$$
a-\lambda=\left[\begin{array}{rr}
-\lambda & 1 \\
-1 & -\lambda
\end{array}\right]
$$

is invertible for every real $\lambda$. Hence we adopt the following definition due to Kaplansky (1949).

Definition 1.1.10 Let $A$ be a real algebra with unit 1. For $a \in A$, the spectrum of $a$ in $A$ is the subset $\operatorname{Sp}(a, A)$ of $\mathbb{C}$ defined as follows:

$$
\operatorname{Sp}(a, A):=\left\{s+i t \in \mathbb{C}:(a-s)^{2}+t^{2} \text { is singular in } A\right\} \text {. }
$$

Clearly, $s+i t \in \operatorname{Sp}(a, A)$ if and only if $s-i t \in \operatorname{Sp}(a, A)$.

Remark 1.1.11 The following facts can be deduced in a straightforward manner from Definitions 1.1.8 and 1.1.10.

(i) If $A$ is a complex algebra with unit and if $A_{R}$ denotes $A$ regarded as a real algebra, then

$$
\operatorname{Sp}\left(a, A_{R}\right)=\operatorname{Sp}(a, A) \cup\{\bar{\lambda}: \lambda \in \operatorname{Sp}(a, A)\} .
$$

(ii) If $A$ is a real algebra with unit and $B$ is the complexification of $A$ (as in Definition 1.1.6), then

$$
\operatorname{Sp}(a, A)=\operatorname{Sp}((a, 0), B) \quad \text { for every } a \text { in } A .
$$

Definition 1.1.12 Let $A$ be a real normed algebra and let $a \in A$. The spectral radius $r(a)$ of $a$ is defined by

$$
r(a):=\inf \left\{\left\|a^{n}\right\|^{1 / n}: n=1,2, \ldots\right\} .
$$

The reason for the nomenclature "spectral radius" will be clear later. Note that $r(a) \leq\|a\|$. Also, if $a b=b a$ for $a, b$ in $A$, then $r(a b) \leq$ $r(a) r(b)$ since $(a b)^{n}=a^{n} b^{n}$ for all $n=1,2, \ldots$

Lemma 1.1.13 Let $A$ be a real normed algebra and let $a \in A$. Then

$$
r(a)=\lim _{n \rightarrow \infty}\left\|a^{n}\right\|^{1 / n}
$$


Proof. By definition, $r(a) \leq\left\|a^{n}\right\|^{1 / n}$ for all $n$. Now let $\varepsilon>0$. Then there exists $k$ such that $\left\|a^{k}\right\|^{1 / k}<r(a)+\varepsilon$. By the division algorithm, for every natural number $n$, there exist unique nonnegative integers $p(n)$ and $q(n)$ such that $n=p(n) k+q(n)$ and $q(n) \leq k-1$. As $n \rightarrow \infty, q(n) / n \rightarrow 0$, hence $p(n) k / n \rightarrow 1$. Thus we have

$$
\left\|a^{n}\right\|^{1 / n} \leq\left\|a^{k}\right\|^{p(n) / n}\|a\|^{q(n) / n} \longrightarrow\left\|a^{k}\right\|^{1 / k}<r(a)+\varepsilon .
$$

This completes the proof.

Now we show that if $a b=b a$, then $r(a+b) \leq r(a)+r(b)$. The following proof, adapted from Rickart (1960), uses Lemma 1.1.13.

Let $\alpha>r(a), \beta>r(b), x=a / \alpha$, and $y=b / \beta$, so that $r(x), r(y)<1$. Since $a b=b a$, we have for $n=1,2, \ldots$,

$$
\begin{aligned}
\left\|(a+b)^{n}\right\|^{1 / n} & =\left\|\sum_{k=0}^{n} C_{k} a^{k} b^{n-k}\right\|^{1 / n} \\
& \leq\left[\sum_{k=0}^{n}{ }_{n} C_{k} \alpha^{k} \beta^{n-k}\left\|x^{k}\right\|\left\|y^{n-k}\right\|\right]^{1 / n} .
\end{aligned}
$$

For each $n$, let $k_{n}$ be such that $0 \leq k_{n} \leq n$ and

$$
\left\|x^{k_{n}}\right\|\left\|y^{n-k_{n}}\right\|=\max _{0 \leq k \leq n}\left\|x^{k}\right\|\left\|y^{n-k}\right\| .
$$

Then it follows that for $n=1,2, \ldots$,

$$
r(a+b) \leq\left\|x^{k_{n}}\right\|^{1 / n}\left\|y^{n-k_{n}}\right\|^{1 / n}(\alpha+\beta) .
$$

Since $0 \leq k_{n} / n \leq 1$, we choose a subsequence $\left(n_{m}\right)$ of natural numbers such that if $k_{m}^{\prime}=k_{n_{m}}$, then $\left(k_{m}^{\prime} / n_{m}\right)$ converges to $\delta$, say. Then $0 \leq \delta \leq 1$. If $\delta=0$, then

$$
\varlimsup_{m \rightarrow \infty}\left\|x^{k_{m}^{\prime}}\right\|^{1 / n_{m}} \leq \varlimsup_{m \rightarrow \infty}\|x\|^{k_{m}^{\prime} / n_{m}}=1,
$$

and if $0<\delta \leq 1$, then $k_{m}^{\prime} \neq 0$ for all large $m$, so that

$$
\varlimsup_{m \rightarrow \infty}\left\|x^{k_{m}^{\prime}}\right\|^{1 / m_{m}}=\varlimsup_{m \rightarrow \infty}\left(\left\|x^{k_{m}^{\prime}}\right\|^{1 / k_{m}^{\prime}}\right)^{k_{m^{\prime}} / n_{m}}=r(x)^{\delta} \leq 1 .
$$

Similarly, $\overline{\lim }_{m \rightarrow \infty}\left\|y^{k_{m}^{\prime}}\right\|^{1 / m_{m}} \leq 1$. Hence $r(a+b) \leq \alpha+\beta$. Since this is true for all $\alpha>r(a)$ and $\beta>r(b)$, we have $r(a+b) \leq r(a)+r(b)$.

Lemma 1.1.14 Let $A$ be a real Banach algebra with unit 1, let $a \in A$, and let $s$ and $t$ be real numbers.

(i) If $r(a)<|s|$, then $a-s$ is regular in $A$ and 


$$
(a-s)^{-1}=-\sum_{n=0}^{\infty} \frac{a^{n}}{s^{n+1}} .
$$

(ii) If $r(a)<\left(s^{2}+t^{2}\right)^{1 / 2}:=q$, then $(a-s)^{2}+t^{2}$ is regular in $A$ and its inverse is given by the absolutely convergent series $\Sigma_{n=0}^{\infty} b_{n}$, where

$$
b_{n}= \begin{cases}\frac{(n+1) a^{n}}{s^{n+2}} & \text { if } t=0, \\ \frac{[\cos n \theta-\cos (n+2) \theta] a^{n}}{(1-\cos 2 \theta) q^{n+2}} & \text { if } t \neq 0, \quad \theta=\tan ^{-1} \frac{t}{s}, \quad 0 \leq \theta<\pi .\end{cases}
$$

Proof.

(i) Let $r(a)<|s|$. There exists $p$ such that $r(a)<p<|s|$. By Lemma 1.1.13, $\left\|a^{n}\right\| \leq p^{n}$ for all sufficiently large $n$. For such $n$, we have

$$
\left\|\frac{a^{n}}{s^{n+1}}\right\| \leq \frac{p^{n}}{|s|^{n+1}}
$$

so that $\sum_{n=0}^{\infty} a^{n} / s^{n+1}$ converges absolutely. Since $A$ is Banach, let

$$
c=-\sum_{n=0}^{\infty} \frac{a^{n}}{s^{n+1}} \quad \text { and } \quad c_{m}=-\sum_{n=0}^{m} \frac{a^{n}}{s^{n+1}} .
$$

Then

$$
\begin{aligned}
c_{m}(a-s) & =-\left(\frac{1}{s}+\frac{a}{s^{2}}+\cdots+\frac{a^{m}}{s^{m+1}}\right)(a-s) \\
& =1-\frac{a^{m+1}}{s^{m+1}}=(a-s) c_{m} .
\end{aligned}
$$

Since $(a / s)^{m}$ tends to zero as $m$ tends to infinity, it follows that

$$
c(a-s)=1=(a-s) c,
$$

that is, $c$ is the inverse of $a-s$.

(ii) Let $r(a)<q=\left(s^{2}+t^{2}\right)^{1 / 2}$. If $t=0$, the conclusion follows from (i). Now let $t \neq 0$, so that $1-\cos 2 \theta \neq 0$. As above, we can find $p$ such that $r(a)<p<q$ and $\left\|a^{n}\right\| \leq p^{n}$ for all large $n$. For such $n$,

$$
\left\|b_{n}\right\| \leq(q|\sin \theta|)^{-2}\left(\frac{p}{q}\right)^{n} \text {. }
$$

Hence $\sum_{n=0}^{\infty} b_{n}$ converges absolutely. Let $d=\Sigma_{n=0}^{\infty} b_{n}$ and $d_{m}=\sum_{n=0}^{m} b_{n}$. Then a tedious but routine computation shows that 


$$
\begin{aligned}
d_{m}\left(a^{2}-2 q a \cos \theta+q^{2}\right)= & 1-\frac{[\cos (m+1) \theta-\cos (m+3) \theta] a^{m+1}}{(1-\cos 2 \theta) q^{m+1}} \\
& +\frac{[\cos m \theta-\cos (m+2) \theta] a^{m+2}}{(1-\cos 2 \theta) q^{m+2}} \\
= & \left(a^{2}-2 q a \cos \theta+q^{2}\right) d_{m} .
\end{aligned}
$$

Since $(a / q)^{m}$ tends to zero as $m$ tends to infinity, we get

$$
d\left[(a-s)^{2}+t^{2}\right]=1=\left[(a-s)^{2}+t^{2}\right] d ;
$$

that is, $d$ is the inverse of $(a-s)^{2}+t^{2}$.

Corollary 1.1.15 Let $A$ be a complex Banach algebra with unit 1. Let $a \in A$ and $\lambda \in \mathbb{C}$. If $r(a)<|\lambda|$, then $a-\lambda$ is regular in $A$ and

$$
(a-\lambda)^{-1}=-\sum_{n=0}^{\infty} \frac{a^{n}}{\lambda^{n+1}} .
$$

Proof. Let $\lambda=s+i t$. If $t=0$, the conclusion follows from the first part of Lemma 1.1.14. Now let $t \neq 0$. Note that $(a-s)^{2}+t^{2}=(a-$ $\lambda)(a-\bar{\lambda})$ is invertible and its inverse is $d=\sum_{n=0}^{\infty} b_{n}$, as given in the second part of the lemma. Thus $(a-\lambda)(a-\bar{\lambda}) d=1=(a-\bar{\lambda}) d(a-$ $\lambda)$. This implies that $(a-\lambda)$ is invertible and its inverse is

$$
(a-\bar{\lambda}) d=\sum_{n=0}^{\infty}(a-s) b_{n}+i \sum_{n=0}^{\infty} t b_{n} .
$$

A simple calculation shows that the coefficient of $a^{n}$ in $\sum_{n=0}^{\infty}(a-s) b_{n}$ is $-[\cos (n+1) \theta] / q^{n+1}$ and the coefficient of $a^{n}$ in $\sum_{n=0}^{\infty} t b_{n}$ is $[\sin (n+1) \theta] /$ $q^{n+1}$, where $q=\left(s^{2}+t^{2}\right)^{1 / 2}=|\lambda|$. hence

$$
(a-\lambda)^{-1}=(a-\bar{\lambda}) d=-\sum_{n=0}^{\infty} \frac{a^{n}}{\lambda^{n+1}} .
$$

Corollary 1.1.16 Let $A$ be a real Banach algebra with unit 1. Suppose that $a \in A$ is invertible and $b \in A$ is such that $\|b-a\| \leq \varepsilon /\left\|a^{-1}\right\|$ with $0 \leq$ $\varepsilon<1$. Then $b$ is invertible and $\left\|b^{-1}-a^{-1}\right\| \leq\left\|a^{-1}\right\|^{2}\|b-a\| /(1-\varepsilon)$.

Proof.

$$
\begin{aligned}
r\left(1-a^{-1} b\right) & \leq\left\|1-a^{-1} b\right\|=\left\|a^{-1} a-a^{-1} b\right\| \\
& \leq\left\|a^{-1}\right\|\|a-b\| \leq \varepsilon<1 .
\end{aligned}
$$


Hence, by (i) of Lemma 1.1.14, $-\left(1-a^{-1} b-1\right)=a^{-1} b$ is regular and we can easily conclude that $b$ is also regular. Further,

$$
\left\|b^{-1}\right\|-\left\|a^{-1}\right\| \leq\left\|b^{-1}-a^{-1}\right\|=\left\|b^{-1}(a-b) a^{-1}\right\| \leq \varepsilon\left\|b^{-1}\right\| .
$$

Hence $\left\|b^{-1}\right\| \leq\left\|a^{-1}\right\| /(1-\varepsilon)$. Thus we have

$$
\begin{aligned}
\left\|b^{-1}-a^{-1}\right\| & \leq\left\|b^{-1}\right\|\|a-b\|\left\|a^{-1}\right\| \\
& \leq \frac{\left\|a^{-1}\right\|^{2}\|a-b\|}{1-\varepsilon} .
\end{aligned}
$$

Remark 1.1.17 Let $A$ be a real Banach algebra with unit 1. Let $\operatorname{Inv}(A)$ denote the set of all invertible elements in $A$. Thus $\operatorname{Inv}(A)$ is a group under multiplication. Corollary 1.1.16 says that $\operatorname{Inv}(A)$ is an open set in $A$ and the map $a \mapsto a^{-1}$ is continuous on $\operatorname{Inv}(A)$. In short, $\operatorname{Inv}(A)$ is a topological group.

Corollary 1.1.18 Let $A$ be a real Banach algebra with unit 1 and $a \in A$. Then $\operatorname{Sp}(a, A)$ is a compact subset of $\mathbb{C}$.

Proof. By Lemma 1.1.14, if $s+i t \in \mathrm{Sp}(a)$, then $\left(s^{2}+t^{2}\right)^{1 / 2} \leq r(a) \leq$ $\|a\|$. Hence $\operatorname{Sp}(a)$ is bounded. Thus it is enough to show that $\operatorname{Sp}(a)$ is closed. We show that its complement in $\mathbb{C}$ is open. Let $s+i t \in \mathbb{C} \backslash \operatorname{Sp}(a)$. Then $(a-s)^{2}+t^{2} \in \operatorname{Inv}(A)$. The map $f: \mathbb{C} \rightarrow A$ defined by $f(x+i y)=$ $(a-x)^{2}+y^{2}$ is continuous and $\operatorname{Inv}(A)$ is an open neighborhood of $f(s+$ $i t)$. Hence there exists an open neighborhood $U$ of $s+i t$ such that $f(U) \subset$ $\operatorname{Inv}(A)$. But then $U \subset \mathbb{C} \backslash \mathrm{Sp}(a)$. Thus $\mathbb{C} \backslash \mathrm{Sp}(a)$ is open.

Theorem 1.1.19 (Spectral radius formula) Let $A$ be a real Banach algebra with unit 1 and $a \in A$. Then

$$
r(a)=\sup \left\{\left(s^{2}+t^{2}\right)^{1 / 2}: s+i t \in \operatorname{Sp}(a, A)\right\} .
$$

In particular, $\operatorname{Sp}(a, A)$ is nonempty.

Proof. Let

$$
\alpha:=\sup \left\{\left(s^{2}+t^{2}\right)^{1 / 2}: s+i t \in \mathrm{Sp}(a, A)\right\} .
$$

Lemma 1.1.14 shows that $r(a) \geq \alpha$. We now prove that $r(a) \leq \alpha$. First, let $r(a)=0$. If $0 \notin \operatorname{Sp}(a, A)$, then $a$ is invertible. Since $a$ and $a^{-1}$ commute, we have $1=r(1)=r\left(a a^{-1}\right) \leq r(a) r\left(a^{-1}\right)=0$, which is absurd. Therefore, $0 \in \operatorname{Sp}(a, A)$, so that $\alpha \geq 0=r(a)$.

Now, let $r(a)>0$ and $\phi$ be a continuous linear functional on $A$. For $s+$ it in $\mathbb{C} \backslash \operatorname{Sp}(a, A)$, we define

$$
u(s, t)=\phi\left[(a-s)\left((a-s)^{2}+t^{2}\right)^{-1}\right]
$$


and

$$
v(s, t)=\phi\left[t\left((a-s)^{2}+t^{2}\right)^{-1}\right] .
$$

It can be verified that $u$ and $v$ both satisfy Laplace's equation and are, in fact, harmonic conjugates of each other in $\mathbb{C} \backslash \operatorname{Sp}(a, A)$. For $s+$ it in $\mathbb{C}$ with $r(a)<\left(s^{2}+t^{2}\right)^{1 / 2}$, we have

$$
u(s, t)=\sum_{n=0}^{\infty} \phi\left[(a-s) b_{n}\right]
$$

and

$$
v(s, t)=\sum_{n=0}^{\infty} \phi\left(t b_{n}\right)
$$

where $b_{n}, n=0,1,2, \ldots$, are as in Lemma 1.1.14. Further, we have already noted in Corollary 1.1.15 that the coefficient of $a^{n}$ in $(a-s) \sum_{n=0}^{\infty}$ $b_{n}$ is $-\cos (n+1) \theta / q^{n+1}$ and in $\sum_{n=0}^{\infty} t b_{n}$ is $\sin (n+1) \theta a^{n} / q^{n+1}$, where $q=$ $\left(s^{2}+t^{2}\right)^{1 / 2}$ and $t / s=\tan \theta, 0 \leq \theta<\pi$. If we let $w=u+i v$ and $z=s+$ $i t$, then

$$
w(z)=-\sum_{n=0}^{\infty} \frac{\phi\left(a^{n}\right)}{z^{n+1}},
$$

where the series converges absolutely for $|z|>r(a)$.

Since $w$ is an analytic function in $\operatorname{C} \backslash \operatorname{Sp}(a, A)$, Laurent's theorem shows that the series above converges for every $z$ with $|z|>\alpha$. For every such $z, \phi\left((a /|z|)^{n}\right)$ is bounded. Since this is true for every continuous linear functional $\phi$ on $A$, by the uniform boundedness principle, there exists $M$ such that $\left\|(a /|z|)^{n}\right\| \leq M$. Hence for every $z$ with $|z|>\alpha$,

$$
r(a)=\lim _{n \rightarrow \infty}\left\|a^{n}\right\|^{1 / n} \leq \lim _{n \rightarrow \infty} M^{1 / n}|z|=|z| .
$$

Thus $r(a) \leq \alpha$.

Remark 1.1.20 The proof above was given in Kulkarni and Limaye (1980). A proof using the complexification technique can be found in Ingelstam (1964), Rickart (1960), and Bonsall and Duncan (1973).

In view of Remark 1.1.11(i), the spectral radius formula is also valid for complex Banach algebras. Note that the spectral radius formula equates an algebraically obtained number $\sup \left\{\left(s^{2}+t^{2}\right)^{1 / 2}: s+i t \in\right.$ $\operatorname{Sp}(a, A)\}$ with $r(a)$, which depends on the norm. This is a good example of the interplay between the algebraic and topological structures of $A$. 
We now give some applications of the spectral radius formula. Let $A$ and $B$ be algebras over a field $F$. Recall that a linear map $T: A \rightarrow B$ is called a homomorphism if $T(a b)=T(a) T(b)$ for all $a, b$ in $A$. A homomorphism is an isomorphism if it is $1: 1$ and onto. $A$ is said to be isomorphic to $B$ if there exists an isomorphism from $A$ onto $B$. Let $X$ and $Y$ be normed linear spaces over $\mathbb{R}$ or $\mathbb{C}$. Then a linear map $S: X \rightarrow Y$ is called an isometry if $\|S(x)\|=\|x\|$ for every $x$ in $X$.

An isometric isomorphism between two normed algebras is a map that is both an isometry and an isomorphism. Whenever such a map exists between two normed algebras, they are called isometrically isomorphic to each other.

Corollary 1.1.21 Let $A$ and $B$ be real Banach algebras with units and $T: A \rightarrow B$ be a homomorphism. Then $r(T(a)) \leq r(a)$ for every $a$ in $A$.

Proof. We denote the units of both $A$ and $B$ by the same symbol 1. It can easily be proved that $T(1)=1$. Now let $s+i t \notin \mathrm{Sp}(a, A)$. Then there is $b$ in $A$ such that

$$
\left[(a-s)^{2}+t^{2}\right] b=1=b\left[(a-s)^{2}+t^{2}\right] .
$$

Hence

$$
\left[(T(a)-s)^{2}+t^{2}\right] T(b)=1=T(b)\left[(T(a)-s)^{2}+t^{2}\right] .
$$

Thus $\operatorname{Sp}(T(a), B) \subset \operatorname{Sp}(a, A)$, and the result follows from 1.1.19.

\section{Remark 1.1.22}

(i) If $T$ is an isomorphism, then $r(T(a))=r(a)$ for every $a$ in $A$.

(ii) Suppose that $B$ has the property $\|b\| \leq k r(b)$ for every $b$ in $B$. A normed algebra satisfying this property is called a spectrally normed algebra. Algebras in Example 1.1.2 and 1.1.4 are spectrally normed. Then

$$
\|T(a)\| \leq k r(T(a)) \leq k r(a) \leq k\|a\|, \quad a \in A .
$$

Thus every homomorphism of $A$ into a spectrally normed Banach algebra is continuous. In particular, every homomorphism of $A$ into $\mathbb{R}$ or $\mathbb{C}$ is continuous. We now prove a fundamental theorem that characterizes normed division algebras. Recall that an algebra with unit is called a division algebra if every nonzero element is regular.

Theorem 1.1.23 (Mazur-Gelfand theorem) Let $A$ be a Banach division algebra.

(i) If $A$ is a complex algebra, then $A$ is isometrically isomorphic to $\mathbb{C}$. 
(ii) If $A$ is a real algebra such that

$$
x, y \in A \text { and } x^{2}+y^{2}=0 \text { imply that } x=y=0,
$$

then $A$ is isometrically isomorphic to R.

(iii) If $A$ is a commutative real algebra and $A$ does not satisfy $\left({ }^{*}\right)$, then $A$ is isomorphic to $\mathbb{C}$.

Proof. In view of Theorem 1.1.19, $\mathrm{Sp}(a, A)$ is nonempty for every $a$ in $A$.

(i) Let $a \in A$ and $\lambda \in \operatorname{Sp}(a, A)$. Then $a-\lambda$ is singular. Hence $a-\lambda=$ 0 . Thus $a=\lambda \cdot 1$ and $\|a\|=|\lambda|\|1\|=|\lambda|$.

(ii) Let $a \in A$ and $s+i t \in \operatorname{Sp}(a, A)$. Then $(a-s)^{2}+t^{2}$ is singular. Hence $(a-s)^{2}+t^{2}=0$. By $\left(^{*}\right)$ this implies that $t=0$ and $a=s$. Also, $\|a\|=|s|$. This proves (ii).

(iii) Since $A$ does not satisfy (*), there exist $x, y$ in $A$ such that $x^{2}+$ $y^{2}=0$ and at least one of (and hence both) $x, y$ are nonzero. Let $j=x y^{-1}$. Then by commutativity $1+j^{2}=0$. Now let $a \in A$ and $s+i t \in \operatorname{Sp}(a, A)$. Then, as above, $(a-s)^{2}+t^{2}=0$, so that

$$
(a-(s+t j))(a-(s-t j))=(a-s)^{2}+t^{2}=0 .
$$

Hence $a=s+t j$ or $a=s-t j$, as $A$ is a division algebra. Thus $A=$ $\operatorname{Span}\{1, j\}$ and $j^{2}=-1$, that is, $A$ is isomorphic to $\mathbb{C}$.

We remark that if $A$ is a real noncommutative Banach division algebra, then $A$ is isomorphic to $H$ (Example 1.1.4): Let $a \in A$ and $s+i t \in$ $\mathrm{Sp}(a, A)$. Then $(a-s)^{2}+t^{2}$ is singular and hence is 0 . This shows that every element of $A$ is a root of a quadratic polynomial with real coefficients. Hence $A$ is finite dimensional by a theorem of Wedderburn. Again, by a theorem of Frobenius, every finite-dimensional real division algebra is isomorphic to $\mathbb{R}, \mathbb{C}$, or $\mathbb{H}$. Since $A$ is noncommutative, $A$ is isomorphic to $H$. Ingelstam (1964) and Bonsall and Duncan (1973) give a proof of this fact without making explicit use of the theorems of Wedderburn and Frobenius.

Let $A$ be a real Banach algebra and $a \in A$. Let $f$ be a complex-valued function defined on some subset of the complex plane. Under certain conditions on $f$, we can define an element in $A$, which can justifiably be denoted by $f(a)$. A study of such conditions and of the relationship between $a$ and $f(a)$ is called functional calculus. We begin this study with polynomials, the simplest functions that one can think of.

Definition 1.1.24 A subset $D$ of $\mathbb{C}$ is said to be symmetric about the real axis if $\bar{z} \in D$ for every $z$ in $D$. 
Let $D \subset \mathbb{C}$ be symmetric about the real axis. By $P_{R}(D)$ we denote the algebra of all polynomials on $D$ with real coefficients. We may note that if $D$ is an infinite set, then a polynomial $p$ with complex coefficients belongs to $P_{R}(D)$ if and only if $p(\bar{z})=\bar{p}(z)$ for every $z$ in $D$.

Let $A$ be a real Banach algebra with unit and let $a \in A$. We have already noted that $\operatorname{Sp}(a, A)$ is symmetric about the real axis. Given $p$ in $P_{R}(D)$ with $p(z)=\alpha_{0}+\alpha_{1} z+\cdots+\alpha_{n} z^{n}, z$ in $D$, we denote by $p(a)$ the element of $A$ given by

$$
p(a)=\alpha_{0}+\alpha_{1} a+\cdots+\alpha_{n} a^{n}
$$

Theorem 1.1.25 Let $A$ be a real Banach algebra with unit $1, a \in A$, and $D$ be an open neighborhood of $\operatorname{Sp}(a, A)$, which is symmetric about the real axis. Then the mapping $p \mapsto p(a)$ is a homomorphism of $P_{R}(D)$ into $A$ which satisfies

$$
\operatorname{Sp}(p(a), A)=\{p(z): z \text { in } \operatorname{Sp}(a, A)\} .
$$

Proof. That $p \mapsto p(a)$ is a homomorphism of $P_{R}(D)$ into $A$ is evident. Now let $\lambda \in \mathbb{C}$, and $p$ in $P_{R}(D)$ be of degree $n$. Then there exist $\beta, \alpha_{1}, \ldots, \alpha_{n}$ in $\mathbb{C}$ such that

$$
\lambda-p(z)=\beta\left(\alpha_{1}-z\right) \cdots\left(\alpha_{n}-z\right) \quad \text { for all } z \text { in } D
$$

Since $z \in D$ implies that $\bar{z} \in D$, replacing $z$ by $\bar{z}$ in (1)

$$
\lambda-p(\bar{z})=\beta\left(\alpha_{1}-\bar{z}\right) \cdots\left(\alpha_{n}-\bar{z}\right) \quad \text { for all } z \text { in } D
$$

Noting that $p(\bar{z})=\overline{p(z)}$ and taking complex conjugates of both sides in (2), we get

$$
\bar{\lambda}-p(z)=\bar{\beta}\left(\bar{\alpha}_{1}-z\right) \cdots\left(\bar{\alpha}_{n}-z\right) .
$$

From (1) and (3), we obtain

$$
(\lambda-p(z))(\bar{\lambda}-p(z))=|\beta|^{2}\left(\alpha_{1}-z\right)\left(\bar{\alpha}_{1}-z\right) \cdots\left(\alpha_{n}-z\right)\left(\bar{\alpha}_{n}-z\right) .
$$

Let $\lambda=s+i t$ and $\alpha_{k}=s_{k}+i t_{k}, k=1,2, \ldots, n$. Then equation (4) becomes

$$
(s-p(z))^{2}+t^{2}=|\boldsymbol{\beta}|^{2}\left[\left(s_{1}-z\right)^{2}+t_{1}^{2}\right] \cdots\left[\left(s_{n}-z\right)^{2}+t_{n}^{2}\right] .
$$

Since $p \mapsto p(a)$ is a homomorphism from $P_{R}(D)$ into $A$, we have

$$
(s-p(a))^{2}+t^{2}=|\beta|^{2}\left[\left(s_{1}-a\right)^{2}+t_{1}^{2}\right] \cdots\left[\left(s_{n}-a\right)^{2}+t_{n}^{2}\right] .
$$

When $p$ is nonconstant, $\beta \neq 0$. Hence $(s-p(a))^{2}+t^{2}$ is singular if and only if $\left(s_{k}-a\right)^{2}+t_{k}^{2}$ is singular for some $k$. Thus $\lambda \in \operatorname{Sp}(p(a))$ if and only if $\lambda=p(z)$ for some $z$ in $\operatorname{Sp}(a)$. 
Let $A, a$, and $D$ be as above. Note that if $p \in P_{R}(D)$ and $p(z) \neq 0$ for all $z$ in $D$, then by Theorem 1.1.25, $p(a)$ is invertible in $A$. By $R_{R}(D)$, we denote the algebra of all rational functions $p / q$, where $p, q \in P_{R}(D)$ and $q(z) \neq 0$ for all $z$ in $D$. For $f=p / q$ in $R_{R}(D)$ by $f(a)$ we denote the element $p(a) q(a)^{-1}$. Then in a similar way we can prove that the mapping $f \mapsto f(a)$ is a homomorphism of $R_{R}(D)$ into $A$, and it satisfies

$$
\operatorname{Sp}(f(a), A)=\{f(z): z \in \operatorname{Sp}(a, A)\} .
$$

We shall now extend the considerations above to a class of functions which is bigger than that of rational functions. A detailed treatment of this topic can be found in Kulkarni and Limaye (1981a). Here we consider only some special cases.

Suppose that $A$ is a real Banach algebra with unit $1, a \in A$, and $\operatorname{Sp}(a) \subset$ $D$, where $D$ is a disk that is symmetric about the real axis. In particular, the center of $D$ is a real number. Let $\alpha$ be the center of $D$, let $\rho$ be the radius of $D$, and $H_{R}(D)$ denote the algebra of all analytic functions $f$ in $D$ satisfying $f(\bar{z})=\bar{f}(z)$ for all $z$ in $D$. Such an $f$ can be expanded in a Taylor series around $\alpha$, so that $f(z)=\sum_{n=0}^{\infty} \lambda_{n}(z-\alpha)^{n}$ for all $z$ in $D$ with $\lambda_{n}$ in $R$ and $\overline{\lim }_{n \rightarrow \infty}\left|\lambda_{n}\right|^{1 / n} \leq 1 / \rho$. Since $\operatorname{Sp}(a) \subset D$, we have $\operatorname{Sp}(a-\alpha) \subset\{z:|z|$ $<\rho\}$, so that $r(a-\alpha)<\eta<\rho$. Hence for large $n,\left\|\lambda_{n}(a-\alpha)^{n}\right\| \leq$ $(\eta / \rho)^{n}$. Since $\Sigma(\eta / \rho)^{n}$ converges, $\Sigma_{n=0}^{\infty} \lambda_{n}(a-\alpha)^{n}$ converges (absolutely) to an element of $A$, which we denote by $f(a)$.

Theorem 1.1.26 (Spectral mapping theorem) Let $A$ be a real Banach algebra with unit $1, a \in A$, and suppose that $\operatorname{Sp}(a) \subset D:=\{z \in$ $\mathbb{C}:|z-\alpha|<\rho\}, \alpha$ in $R$. Then the mapping $f \mapsto f(a)$ is a homomorphism of $H_{R}(D)$ into $A$, satisfying

$$
\operatorname{Sp}(f(a), A)=\{f(z): z \in \mathrm{Sp}(a, A)\} .
$$

Proof. That the mapping $f \mapsto f(a)$ is a homomorphism is obvious. Now let $f \in H_{R}(D)$,

$$
f(z)=\sum_{n=0}^{\infty} \lambda_{n}(z-\alpha)^{n}, \quad p_{m}(z):=\sum_{n=0}^{\infty} \lambda_{n}(z-\alpha)^{n}, \quad m=0,1,2, \ldots
$$

$\lambda_{n}$ in $\Omega, z$ in $D$. Then $p_{m} \in P_{R}(D)$ for all $m=0,1,2, \ldots,\left\|p_{m}(a)-f(a)\right\| \rightarrow$ 0 , and $p_{m}$ converges uniformly to $f$ over each closed disk contained in $D$.

Let $\lambda \in \operatorname{Sp}(a), f(\lambda)=s+i t$, and $p_{m}(\lambda)=s_{m}+i t_{m}$. Since $p_{m}(a) \rightarrow$ $f(a)$ and $s_{m}+i t_{m} \rightarrow s+i t$, we have $\left(s_{m}-p_{m}(a)\right)^{2}+t_{m}^{2} \rightarrow(s-f(a))^{2}+t^{2}$ as $m \rightarrow \infty$. If $s+i t \notin \mathrm{Sp}(f(a))$, then $(s-f(a))^{2}+t^{2}$ is invertible and hence $\left(s_{m}-p_{m}(a)\right)^{2}+t_{m}^{2}$ is invertible for large $m$, by Remark 1.1.17. 
Hence for such $m, p_{m}(\lambda)=s_{m}+i t_{m} \notin \operatorname{Sp}\left(p_{m}(a)\right)$. This contradicts Theorem 1.1.25. Hence $\{f(z): z \in \operatorname{Sp}(a)\} \subset \operatorname{Sp}(f(a))$.

Next, let $z \in \operatorname{Sp}(f(a))$. Then $f(\operatorname{Sp}(a)):=\{f(\lambda): \lambda \in \operatorname{Sp}(a)\}$ is a compact set by Corollary 1.1.18. If $z \notin f(\operatorname{Sp}(a))$, then

$$
\delta:=\inf \{|z-f(\lambda)|: \lambda \in \operatorname{Sp}(a)\}>0 .
$$

Again $\mathrm{Sp}(a)$, being compact, is contained in a closed disk contained in $D$, and $p_{n} \rightarrow f$ uniformly over each such disk. Hence we can find $m_{0}$ such that for all $m \geq m_{0}$ and $\lambda \in \operatorname{Sp}(a)$, we have $\left|f(\lambda)-p_{m}(\lambda)\right|<\delta / 2$. But then, for all $\lambda$ in $\operatorname{Sp}(a)$ and $m \geq m_{0}$,

$$
\left|z-p_{m}(\lambda)\right| \geq|z-f(\lambda)|-\left|f(\lambda)-p_{m}(\lambda)\right| \geq \frac{\delta}{2}>0 .
$$

In view of Theorem 1.1.25, this implies that $z \notin \operatorname{Sp}\left(p_{m}(a)\right)$ for all $m \geq$ $m_{0}$. Let $z=s+i t$ and $b_{m}=\left(s-p_{m}(a)\right)^{2}+t^{2}$. Then $b_{m}$ is invertible for all $m \geq m_{0}$. Further, by the comment following Theorem 1.1.25, we have for $m \geq m_{0}$,

$$
\begin{aligned}
& r\left(b_{m}^{-1}\right)=\sup \left\{\left|\left(\left(s-p_{m}(\lambda)\right)^{2}+t^{2}\right)^{-1}\right|: \lambda \in \operatorname{Sp}(a)\right\} \\
& =\sup \left\{\left|z-p_{m}(\lambda)\right|^{-1}\left|\bar{z}-p_{m}(\lambda)\right|^{-1}: \lambda \in \operatorname{Sp}(a)\right\} \\
& \leq \frac{4}{\delta^{2}} \text {. }
\end{aligned}
$$

Now, let $b=(s-f(a))^{2}+t^{2}$. Then $b_{m} \rightarrow b$ as $m \rightarrow \infty$. Hence we can find $m \geq m_{0}$ such that $\left\|b_{m}-b\right\|<\delta^{2} / 4$. Note that $b_{m}$ commutes with $b$. Hence the comment after Definition 1.1.12 shows that

$$
\begin{aligned}
r\left(1-b_{m}^{-1} b\right)=r\left(b_{m}^{-1}\left(b_{m}-b\right)\right) & \leq r\left(b_{m}^{-1}\right) r\left(b_{m}-b\right) \\
& \leq \frac{4}{\delta^{2}}\left\|b_{m}-b\right\|<1 .
\end{aligned}
$$

By Lemma $1.1 .14, b_{m}^{-1} b$ is invertible and hence $b$ is invertible. This contradiction implies that

$$
\operatorname{Sp}(f(a)) \subset f(\operatorname{Sp}(a)) .
$$

Remark 1.1.27 Let $A$ be a real Banach algebra with unit 1. Since $\exp (z)$ is an entire function and $\Sigma_{n=0}^{\infty} z^{n} / n$ ! converges uniformly to $\exp (z)$ over every closed disk with center at 0 , we have $\exp (a)=\sum_{n=0}^{\infty} a^{n} / n !$. One can show that if $a$ and $b$ commute, then $\exp (a+b)=\exp (a) \exp (b)$. Hence $\exp (a)$ is invertible and its inverse is $\exp (-a)$. Now let

$$
\begin{aligned}
\mathbb{C}^{+}: & =\{s+i t \in \mathbb{C}: s>0 \text { or } t \neq 0\} \\
& =\mathbb{C} \backslash\{t \in \mathbb{R}: t \leq 0\} .
\end{aligned}
$$


For $z$ in $\mathbb{C}^{+}, z=r \exp (i \theta), r>0,-\pi<\theta<\pi$. We denote by $\operatorname{Ln}(z)$ the principal branch of the logarithm of $z$, that is, $\operatorname{Ln}(z)=\ln r+i \theta,-\pi$ $<\theta<\pi$. Then

$$
\operatorname{Ln}(\exp (z))=z \quad \text { for all } z \text { in } \mathbb{C}
$$

and

$$
\exp (\operatorname{Ln}(z))=z \quad \text { for all } z \text { in } \mathbb{C}^{+} .
$$

Thus, by Theorem 1.1.26, if $\operatorname{Sp}(a) \subset\{z \in \mathbb{C}:|z-\alpha|<\rho\} \subset \mathbb{C}^{+}$for some $\alpha$ in $R$, then $\operatorname{Ln}(a) \in A$ and

$$
\exp (\operatorname{Ln}(a))=a, \quad \operatorname{Ln}(\exp (a))=a .
$$

Corollary 1.1.28 Let $A$ be a real Banach algebra with unit 1 and let $a \in A$. If $r(1-a)<1$, then

$$
\operatorname{Ln}(a)=-\sum_{n=1}^{\infty} \frac{(1-a)^{n}}{n} \in A .
$$

Proof. Since $r(1-a)<1, \operatorname{Sp}(a) \subset\{z \in \mathbb{C}:|z-1|<1\} \subset \mathbb{C}^{+}$. Hence $\operatorname{Ln}(a) \in A$. Also, the Taylor series expansion of $\operatorname{Ln}(z)$ around 1 is $\operatorname{Ln}(z)=-\sum_{n=1}^{\infty}(1-z)^{n} / n$. Hence the result.

We shall now give a few criteria for the commutativity of a Banach algebra. These will be used in Section 1.4. The following lemma is crucial in the proof of these criteria.

Lemma 1.1.29 Let $A$ be a real or complex Banach algebra with unit 1 . Then:

(i) $\mathrm{Sp}(a b) \backslash\{0\}=\mathrm{Sp}(b a) \backslash\{0\}$ for all $a, b$ in $A$, so that $r(a b)=r(b a)$.

(ii) If there exists $k>0$ such that $\|a\|^{2} \leq k\left\|a^{2}\right\|$ for all $a$ in $A$, then $A$ is a spectrally normed algebra, in fact; $\|a\| \leq k r(a)$ for all $a$ in $A, r(a b)=$ $r(b a)$, and $\|b a\| \leq k\|a b\|$ for all $a, b$ in $A$.

Proof.

(i) First, let $A$ be a real algebra. Let $s+i t$ be in $C, s^{2}+t^{2}=1$, and $s+i t$ be not in $\operatorname{Sp}(a b)$. Let $c$ denote the inverse of $(a b-s)^{2}+t^{2}$. Then it follows by direct calculation that $1+b(2 s-a b) c a$ is the inverse of $(b a-s)^{2}+t^{2}$, so that $s+i t \notin \mathrm{Sp}(b a)$. If $A$ is a complex algebra, one can give a similar proof, or use Remark 1.1.11(i). The spectral radius formula (Theorem 1.1.19) shows that $r(a b)=r(b a)$.

(ii) Since $\|a\|^{2} \leq k\left\|a^{2}\right\|$ for all $a$ in $A$, we have by induction 


$$
\|a\|^{2^{n}} \leq k^{2^{n}-1}\left\|a^{2^{n}}\right\| \quad \text { for all } n=1,2, \ldots
$$

Hence $\|a\| \leq k r(a)$ by taking $2^{n}$ th roots and applying Lemma 1.1.13. Now it follows from (i) that

$$
\|b a\| \leq k r(b a)=k r(a b) \leq k\|a b\| .
$$

Theorem 1.1.30 Suppose that $A$ is a Banach algebra with unit 1 satisfying one of the following conditions:

(i) (Le Page, 1967; Hirschfeld and Zelazko, 1968) $A$ is a complex algebra and there exists a positive constant $k$ such that $\|a\|^{2} \leq k\left\|a^{2}\right\|$ for all $a$ in $A$.

(ii) (Kulkarni, 1988; Srivastav, 1990) $A$ is a real algebra and there exists a positive constant $k$ such that $\|a\|^{2} \leq k\left\|a^{2}+b^{2}\right\|$ for all $a, b$ in $A$ with $a b=b a$.

Then $A$ is commutative.

Proof. In both cases $\|a\|^{2} \leq k\left\|a^{2}\right\|$ for all $a$ in $A$. Hence by (ii) of Lemma 1.1.29,

$$
\|b a\| \leq k\|a b\| \quad \text { for all } a, b \text { in } A .
$$

Now let $\phi$ be a continuous linear functional on $A$, and let $a$ and $b$ be elements of $A$.

Suppose that $A$ satisfies condition (i). Define $f: \mathbb{C} \rightarrow \mathbb{C}$ by

$$
f(z):=\phi[\exp (z a) b \exp (-z a)]
$$

for $z$ in $\mathbb{C}$. Then $f$ is an entire function. Further, by (1),

$$
\begin{aligned}
|f(z)| & \leq\|\phi\|\|\exp (z a) b \exp (-z a)\| \\
& \leq k\|\phi\|\|b \exp (-z a) \exp (z a)\|=k\|\Phi\|\|b\| .
\end{aligned}
$$

Thus $f$ is bounded and hence it is a constant by Liouville's theorem. Therefore, $f(0)=f(1)$ implies that

$$
\phi(b)=\phi[\exp (a) b \exp (-a)] .
$$

Next, suppose that $A$ satisfies condition (ii). Define $u: \mathbb{R}^{2} \rightarrow \mathbb{R}^{1}$ by $u(x, y)=\phi\{\exp (x a)[(\cos y a) b \cos y a+(\sin y a) b \sin y a] \exp (-x a)\}$ for $x, y$ in $\mathbb{R}^{2}$.

A straightforward computation of partial derivatives shows that $u$ is a harmonic function on $\Omega^{2}$. (The details of this computation can be found in the references cited above.) Also, for all $x, y$ in $\Omega^{2}$, we have by (1), 


$$
\begin{aligned}
|u(x, y)| & \leq\|\phi\|\|\exp (x a)[(\cos y a) b \cos y a+(\sin y a) b \sin y a] \exp (-x a)\| \\
& \leq k\|\phi\|\|(\cos y a) b \cos y a+(\sin y a) b \sin y a\| \\
& \leq k\|\phi\|\|b\|\left(\|\cos y a\|^{2}+\|\sin y a\|^{2}\right) \\
& \leq 2 k^{2}\|\phi\|\|b\|\left\|\cos ^{2} y a+\sin ^{2} y a\right\| \\
& =2 k^{2}\|\phi\|\|b\| .
\end{aligned}
$$

Thus $u$ is a bounded harmonic function on $\Omega^{2}$. Hence $u$ is a constant. Therefore, $u(0,0)=u(1,0)$ again implies equation (2).

Since equation (2) holds for every continuous linear functional $\phi$ on $A$, we have

$$
b=\exp (a) b \exp (-a)
$$

that is,

$$
b \exp (a)=\exp (a) b \quad \text { for all } a, b \text { in } A .
$$

Now let $c$ and $d$ be any two elements of $A$. If $\|c\|<1$, there exists $a$ in $A$ such that $1+c=\exp (a)$ (Corollary 1.1.28). From (3) it follows that $d \exp (a)=\exp (a) d$; that is, $d(1+c)=(1+c) d$, or $c d=d c$. The restriction $\|c\|<1$ can now be removed by considering $c / 2\|c\|$ if $c \neq 0$. $\square$

Example 1.1.4 shows that if unlike in part (i) of Theorem 1.1.30, $A$ is a real Banach algebra satisfying $\|a\|^{2} \leq k\left\|a^{2}\right\|$ for all $a$ in $A$ and some positive content $k$, then $A$ need not be commutative.

\subsection{COMMUTATIVE REAL BANACH ALGEBRAS}

We now proceed to develop the Gelfand theory for real commutative Banach algebras. The aim of this theory is to show that every real commutative Banach algebra with unit can be represented as an algebra of continuous functions on a compact Hausdorff space. The main theorem in this direction (Theorem 1.2.9) is due to Ingelstam (1964).

Definition 1.2.1 Let $A$ be a real algebra. The carrier space of $A$, denoted by $\operatorname{Car}(A)$, is the set of all nonzero homomorphisms from $A$ to $\mathbb{C}$, regarded as a real algebra.

Let $\phi \in \operatorname{Car}(A)$ and define $\bar{\phi}$ by $\bar{\phi}(a)=\overline{\phi(a)}$ for $a$ in $A$. Then it is easy to see that $\bar{\phi} \in \operatorname{Car}(A)$. Let $\tau: \operatorname{Car}(A) \rightarrow \operatorname{Car}(A)$ be defined by $\tau(\phi)=\bar{\phi}, \phi$ $\in \operatorname{Car}(A)$.

For $a$ in $A$, the Gelfand transform of $a$ is the map $\hat{a}: \operatorname{Car}(A) \rightarrow \mathbb{C}$, given by $\hat{a}(\phi)=\phi(a)$ for $\phi$ in $\operatorname{Car}(A)$. The weakest topology on $\operatorname{Car}(A)$ that makes $\hat{a}$ continuous on $\operatorname{Car}(A)$ for all $a$ in $A$ is called the Gelfand topology on $\operatorname{Car}(A)$. 
Note that if $A$ has unit 1 , and $\phi \in \operatorname{Car}(A)$, then $\phi(1)=1$, because $\phi$ is a nonzero homomorphism. If $a$ is invertible, then $\phi\left(a^{-1}\right) \phi(a)=1$. Thus $\phi(a)$ is nonzero for every invertible element $a$.

Lemma 1.2.2 Let $A$ be a real Banach algebra with unit 1 and $\phi \in$ $\operatorname{Car}(A)$. Then $\|\phi\|:=\sup \{|\phi(a)|: a \in A, \quad\|a\| \leq 1\}=1$.

Proof. By Corollary 1.1.21, $|\phi(a)| \leq\|a\|$ for all $a$ in $A$. Hence $\|\phi\| \leq$ 1. Since $\phi(1)=1$, we have $\|\phi\|=1$.

The following example shows that $\operatorname{Car}(A)$ can, in general, be empty. Consider the real quaternion algebra $H$ of Example 1.1.4. Suppose that $\phi$ $\in \operatorname{Car}(\because)$. Then

$$
\begin{aligned}
\phi(2 k) & =\phi(i j-j i) \\
& =\phi(i) \phi(j)-\phi(j) \phi(i)=0 .
\end{aligned}
$$

Since $2 k$ is invertible, we have $1=\phi(1)=\phi(2 k) \phi\left((2 k)^{-1}\right)=0$, a contradiction. Hence Car(M) is empty. We shall, however, show in the sequel that if $A$ is a commutative normed algebra, then $\operatorname{Car}(A)$ is nonempty.

Definition 1.2.3 Let $A$ be a real or complex algebra with unit. A subspace $I$ of $A$ is called a left ideal if $a \in I$ and $x \in A$ imply that $x a \in I$. Similarly, a right ideal is defined. $I$ is called a (two-sided) ideal if $I$ is both a left and a right ideal. An ideal (left, right, two-sided) is called proper if it is different from $A$. When $A$ is commutative, every left or right ideal is two-sided. A maximal ideal is a proper ideal $M$ which is not contained in any other proper ideal; that is, if $I$ is an ideal and $M \subset I \subset A$, then $M=I$ or $I=A$. By a routine application of Zorn's lemma, one can prove that every (left, right, two-sided) proper ideal is contained in a maximal (respectively, left, right, two-sided) ideal.

Lemma 1.2.4 Let $I$ be a closed two-sided ideal in a (real or complex) normed algebra $A$ with unit. Let $A / I$ denote the set of all cosets of the form $I+a:=\{x+a: x \in I\}$. For $a, b$ in $A$ and $\alpha$ in R (or $\mathbb{C}$ ), define

$$
\begin{aligned}
(I+a)+(I+b) & =I+(a+b), \\
(I+a) \cdot(I+b) & =I+a b,
\end{aligned}
$$

and

$$
\alpha(I+a)=I+\alpha a .
$$

Then the operations above are well defined and under these operations $A / I$ 
is an algebra with unit $I+1$. Further, define $\|I+a\|:=\inf \{\|x+a\|$ : $x \in I\}$ for $a$ in $A$. Then $A / I$ is a normed algebra with respect to this norm. If $A$ is Banach, then $A / I$ is Banach.

Proof. Straightforward.

Lemma 1.2.5 Let $I$ be a proper ideal in a (real or complex) commutative Banach algebra $A$ with unit 1 . Then the closure $J$ of $I$ is a proper ideal. In particular, every maximal ideal is closed.

Proof. Clearly, $J$ is an ideal. If $a \in I$ and $\|1-a\|<1$, then $a$ is invertible by Lemma 1.1.14. Thus $\|1-a\| \geq 1$ for every $a$ in $I$ and hence for every $a$ in $J$. Hence $1 \notin J$, so that $J$ is proper.

Theorem 1.2.6 Let $A$ be a real commutative Banach algebra with unit 1.

(i) If $\phi \in \operatorname{Car}(A)$, then the kernel of $\phi$, denoted by ker $\phi:=\{a \in A: \phi(a)$ $=0\}$, is a maximal ideal in $A$.

(ii) If $M$ is a maximal ideal in $A$, then $M=\operatorname{ker} \phi$ for some $\phi$ in $\operatorname{Car}(A)$.

(iii) For $\phi, \psi$ in $\operatorname{Car}(A)$, if $\operatorname{ker} \phi=\operatorname{ker} \psi$, then $\psi=\phi$ or $\psi=\bar{\phi}$.

Proof.

(i) If $a \in \operatorname{ker} \phi, b \in A$, then $\phi(a b)=\phi(a) \phi(b)=0$. Thus $\operatorname{ker} \phi$ is an ideal. Since $\phi(1)=1, \operatorname{ker} \phi$ is proper. Suppose that $\operatorname{ker} \phi$ is properly contained in an ideal $I$. We consider two cases to show that $I=A$.

Case (a): Suppose that $\phi(A) \subset R$. Then we can find $b$ in $I$ such that $\phi(b)=s \neq 0, s \in$ R. Let $a \in A$. If $c=a-\phi(a) b / s$, then $\phi(c)=0$. Hence $c \in \operatorname{ker} \phi \subset I$. Thus $a=c+\phi(a) b / s \in I$. This shows that $I=A$.

Case (b): If $\phi(A)$ is not contained in R, we can find $b$ in $A$ such that $\phi(b)=s+i t$ and $t \neq 0$. Let $c=(b-s) / t$. Then $\phi(c)=i$. There exists $d$ in $I$ such that $\phi(d)=p+i q \neq 0, p, q$ in 圆. Let $e=d\left(-p c^{2}-q c\right) /$ $\left(p^{2}+q^{2}\right)$. Then $e \in I$ and $\phi(e)=1$. Now let $a \in A$ and $\phi(a)=x+i y$. Then $a-x e-y c e \in \operatorname{ker} \phi \subset I$. Also, $x e+y c e \in I$. Hence $a \in I$. This shows that $I=A$.

(ii) Let $M$ be a maximal ideal. Then $M$ is closed by Lemma 1.2.5. Lemma 1.2.4 shows that $A / M$ is a real commutative Banach algebra with unit. Since $M$ is maximal, it is easy to prove that $A / M$ is a division algebra. Hence by the Mazur-Gelfand theorem 1.1.23, $A / M$ is isomorphic to 同 or $\mathbb{C}$. Let $\theta$ be such an isomorphism and let $\pi: A \rightarrow A / M$ be the canonical map. Then $\phi:=\theta \circ \pi \in \operatorname{Car}(A)$ and $\operatorname{ker} \phi=M$.

(iii) Let $\phi, \psi \in \operatorname{Car}(A)$ and $\operatorname{ker} \phi=\operatorname{ker} \psi=M$. Let $\pi: A \rightarrow A / M$ be the canonical map and $\tilde{\phi}$ and $\tilde{\psi}$ be defined by $\tilde{\phi}((M+a)):=\phi(a)$ and $\tilde{\psi}((M+a)):=\psi(a)$ for $a$ in $A$. Then $\tilde{\phi}$ and $\tilde{\psi}$ are both isomorphisms 
from $A / M$ to $\Omega$ or $C$. Hence $\tilde{\phi} \circ(\tilde{\psi})^{-1}$ is an isomorphism from $R$ to $R$ or from $\mathbb{C}$ to $\mathbb{C}$. The only isomorphism from $R$ to $R$ is the identity map and the only isomorphisms from $\mathbb{C}$ to $\mathbb{C}$, where $\mathbb{C}$ is regarded as a real algebra, are (i) the identity map and (ii) the complex conjugation, that is, the map $z \mapsto \bar{z}$. This implies that $\tilde{\psi}=\tilde{\phi}$ or $\tilde{\psi}=\tilde{\phi}$. Since $\phi=\tilde{\phi} \circ \pi$ and $\psi=$ $\tilde{\psi} \circ \pi$, we have $\psi=\phi$ or $\psi=\bar{\phi}$.

Remark 1.2.7 The set of all maximal ideals of a real commutative Banach algebra $A$ with unit is denoted by $M(A)$ and is called the maximal ideal space of $A$. Theorem 1.2.6 shows that the map $\operatorname{ker}: \operatorname{Car}(A) \rightarrow M(A)$ (i.e., the map $\phi \mapsto \operatorname{ker} \phi)$ is onto. This implies that $\operatorname{Car}(A)$ is nonempty. Further, for $M$ in $M(A)$, the inverse image $\operatorname{ker}^{-1}(M)$ consists of $\{\phi, \bar{\phi}\}$ for some $\phi$ in $\operatorname{Car}(A)$.

Remark 1.2.8 Let $A$ be a complex commutative Banach algebra with unit 1 . The carrier space of $A$, denoted by $\operatorname{Car}(A)$, is the set of all nonzero homomorphisms from $A$ to $\mathbb{C}$, where $\mathbb{C}$ is regarded as a complex algebra. Hence if $\phi \in \operatorname{Car}(A)$, then $\phi(i)=i$. Let $A_{R}$ denote $A$, regarded as a real algebra. It is easy to see that if $\phi \in \operatorname{Car}(A)$, then $\phi$ as well as $\bar{\phi}$ are in $\operatorname{Car}\left(A_{R}\right)$. On the other hand, if $\phi \in \operatorname{Car}\left(A_{R}\right)$, then since $\phi(i)^{2}=-1, \phi(i)$ $= \pm i$. Thus exactly one of $\phi$ and $\phi$ belongs to $\operatorname{Car}(A)$ : that is, $\operatorname{Car}\left(A_{R}\right)=$ $\operatorname{Car}(A) \cup\{\bar{\phi}: \phi \in \operatorname{Car}(A)\}$. In other words, $\operatorname{Car}\left(A_{R}\right)$ is a disjoint union of two copies of $\operatorname{Car}(A)$. Hence if $\phi \in \operatorname{Car}(A)$, then $\|\phi\|=1$ by Lemma 1.2.2. Further, it is easy to see that the ideals of $A$ and $A_{R}$ are the same. Hence $M(A)=M\left(A_{R}\right)$. Thus statements (i) and (ii) of Theorem 1.2.6 remain valid for a complex algebra. As for statement (iii), by Remark 1.2.7, for $M$ in $M(A), \operatorname{ker}^{-1}(M)$ contains the points $\phi$ and $\bar{\phi}$ in $\operatorname{Car}\left(A_{R}\right)$. But exactly one of these is in $\operatorname{Car}(A)$. Thus for complex algebras, statement (iii) should be replaced by:

(iii) $^{\prime}$ If for $\phi, \psi$ in $\operatorname{Car}(A)$, $\operatorname{ker} \phi=\operatorname{ker} \psi$, then $\psi=\phi$.

Hence the map ker: $\operatorname{Car}(A) \rightarrow M(A)$ is a bijection. This map is often used to identify the carrier space and the maximal ideal space of a complex commutative Banach algebra with unit element.

We have now developed sufficient machinery to be able to present the main theorem.

Theorem 1.2.9 (Ingelstam) Let $A$ be a real commutative Banach algebra with unit 1 . Then

(i) $\operatorname{Car}(A)$, endowed with the Gelfand topology, is a compact Hausdorff space. 
(ii) The map $\tau$ : $\operatorname{Car}(A) \rightarrow \operatorname{Car}(A)$, defined by $\tau(\phi)=\Phi$, is a homeomorphism and $\tau \circ \tau$ is the identity map on $\operatorname{Car}(A)$.

(iii) The set of all fixed points of $\tau$ is closed in $\operatorname{Car}(A)$.

(iv) The mapping $a \mapsto \hat{a}, a \in A$, is a homomorphism of $A$ into $\mathrm{C}(\operatorname{Car}(A))$, the algebra of all complex-valued continuous functions on $\operatorname{Car}(A)$.

(v) $a$ is singular in $A$ if and only if $\hat{a}(\phi)=0$ for some $\phi$ in $\operatorname{Car}(A)$.

(vi) For $a \in A, \hat{a}(\operatorname{Car}(A))=\operatorname{Sp}(a, A)$.

(vii) $\|\hat{a}\|:=\sup \{|\hat{a}(\phi)|: \phi \in \operatorname{Car}(A)\}=r(a)$.

Proof.

(i) Let $\phi, \psi \in \operatorname{Car}(A)$ and $\phi \neq \psi$. This means that for some $a$ in $A, \phi(a)$ $\neq \psi(a)$; that is, $\hat{a}(\phi) \neq \hat{a}(\psi)$. Since $\hat{a}$ is continuous with respect to the Gelfand topology, we can enclose $\phi$ and $\psi$ in two disjoint open sets, namely,

$$
N_{\phi}:=\{\theta \in \operatorname{Car}(A):|\hat{a}(\phi)-\hat{a}(\theta)|<\varepsilon\}
$$

and

$$
N_{\psi}:=\{\theta \in \operatorname{Car}(A):|\hat{a}(\psi)-\hat{a}(\theta)|<\varepsilon\},
$$

with $0<\varepsilon<|\phi(a)-\psi(a)| 2$. Thus $\operatorname{Car}(A)$ is Hausdorff. To prove the compactness, we proceed as follows. For each $a$ in $A$, let $K_{A}:=\{z \in$ $\mathbb{C}:|z| \leq\|a\|\}$ and $K:=\Pi_{a \in A} K_{a}$ be the topological product. Since each $K_{a}$ is compact (with the usual topology in (G), $K$ is compact by Tychonoff's theorem [see Simmons (1963)]. We have observed in Lemma 1.2.2 that for $\phi$ in $\operatorname{Car}(A)$ and $a$ in $A,|\phi(a)| \leq\|a\|$. Thus $\phi(a) \in K_{a}$. Hence $\phi$ can be considered as a point of $K$. Consequently, $\operatorname{Car}(A)$ can be considered as a subset of $K$. Further, it is clear that the Gelfand topology on $\operatorname{Car}(A)$ is the same as the (relative) topology of $\operatorname{Car}(A)$ as a subspace of $K$. Thus to prove the compactness of $\operatorname{Car}(A)$, it is sufficient to show that $\operatorname{Car}(A)$ is closed in $K$. Let $\psi \in K$ be in the closure of $\operatorname{Car}(A)$. Since $\psi \in K, \psi$ is a function on $A$ such that $|\psi(a)| \leq\|a\|$ for each $a$ in $A$. Since $\psi$ is in the closure of $\operatorname{Car}(A)$, there is a net $\left\{\psi_{\alpha}\right\}$ in $\operatorname{Car}(A)$ converging to $\psi$. This means that $\psi_{\alpha}(a) \rightarrow \psi(a)$ for every $a$ in $A$. Hence, for $a, b$ in $A$,

$$
\psi(a+b)=\lim \psi_{\alpha}(a+b)=\lim \left(\psi_{\alpha}(a)+\psi_{\alpha}(b)\right)=\psi(a)+\psi(b) .
$$

Similarly, $\psi(s a)=s \psi(a), \psi(a b)=\psi(a) \psi(b)$, and $\psi(1)=1$ for $a, b$ in $A$, $s$ in R. Thus $\psi \in \operatorname{Car}(A)$.

(ii) Clearly, $(\tau \circ \tau)(\phi)=\tau(\bar{\phi})=\phi$ for all $\phi$ in $\operatorname{Car}(A)$. Now suppose that a net $\left\{\phi_{\alpha}\right\}$ converges to $\phi$ in $\operatorname{Car}(A)$. Then for each $a$ in $A, \hat{a}\left(\phi_{\alpha}\right) \rightarrow$ $\hat{a}(\phi)$. Hence $\phi_{\alpha}(a) \rightarrow \phi(a)$, or $\overline{\phi_{\alpha}(a)} \rightarrow \overline{\phi(a)}$; that is, $\hat{a}\left(\tau\left(\phi_{\alpha}\right)\right) \rightarrow \hat{a}(\tau(\phi))$. This means that $\tau\left(\phi_{\alpha}\right) \rightarrow \tau(\phi)$. Thus $\tau$ is continuous. Since $\tau^{-1}=\tau$, $\tau$ is a homeomorphism. 
(iii) Follows from (ii).

(iv) Let $a, b \in A$ and $s \in$ R. For each $\phi$ in $\operatorname{Car}(A)$, we have $\phi(a+b)=$ $\phi(a)+\phi(b)$; that is, $(a+b)^{\hat{\imath}}=\hat{a}+\hat{b}$. Similarly, $(s a)^{\hat{\prime}}=s \hat{a}$ and $(a b)^{\hat{n}}=\hat{a} \hat{b}$.

(v) If $a$ is invertible, then $\phi(a) \phi\left(a^{-1}\right)=1$. Hence $\phi(a) \neq 0$ for every $\phi$ in $\operatorname{Car}(A)$. If $a$ is a singular, then $I:=\{a b: b \in A\}$ is a proper ideal. $I$ is contained in a maximal ideal $M$. By Theorem 1.2.6, $M=\operatorname{ker} \phi$ for some $\phi$ in $\operatorname{Car}(A)$. For this $\phi, \phi(a)=0=\hat{a}(\phi)$.

(vi) $s+i t \in \hat{a}(\operatorname{Car}(A))$ if and only if $s+i t=\phi(a)$ for some $\phi \epsilon$ $\operatorname{Car}(A)$ if and only if $\phi\left((a-s)^{2}+t^{2}\right)=0$ for some $\phi$ in $\operatorname{Car}(A)$ if and only if $(a-s)^{2}+t^{2}$ is singular in $A$ if and only if $s+i t \in \operatorname{Sp}(a, A)$.

(vii) Follows from (vi) and the spectral radius formula (Theorem 1.1.19).

Remark 1.2.10 Let $A$ be a complex commutative Banach algebra with unit 1 . For $a \in A$, the Gelfand transform $\hat{a}$ of $a$ is defined by $\hat{a}(\phi)=\phi(a)$ for $\phi$ in $\operatorname{Car}(A)$. The weakest topology on $\operatorname{Car}(A)$ that makes $\hat{a}$ continuous for each $a$ in $A$ is called the Gelfand topology on $\operatorname{Car}(A)$. Then:

(i) $\operatorname{Car}(A)$ is a compact Hausdorff space with respect to the Gelfand topology.

(ii) The mapping $a \mapsto \hat{a}$ is a homomorphism of $A$ into $C(\operatorname{Car}(A))$.

(iii) $a$ is singular in $A$ if and only if $a(\phi)=0$ for some $\phi$ in $\operatorname{Car}(A)$.

(iv) $\hat{a}(\operatorname{Car}(A))=\operatorname{Sp}(a, A)$ for each $a$ in $A$.

(v) $\|\hat{a}\|:=\sup \{|\hat{a}(\phi)|: \phi \in \operatorname{Car}(A)\}=r(a)$.

All the statements above can be proved easily from Theorem 1.2 .9 by noting the following facts. If $A_{R}$ denotes $A$, regarded as a real algebra, then by Remark 1.2.8,

$$
\operatorname{Car}\left(A_{R}\right)=\operatorname{Car}(A) \cup\{\bar{\phi}: \phi \in \operatorname{Car}(A)\} .
$$

For $a$ in $A$, the Gelfand transform $\hat{a}$ is the restriction to $\operatorname{Car}(A)$ of the Gelfand transform of $a$ as an element of $A_{R}$. The latter is defined on $\operatorname{Car}\left(A_{R}\right)$. Also, by Remark 1.1.11,

$$
\operatorname{Sp}\left(a, A_{R}\right)=\operatorname{Sp}(a, A) \cup\{\bar{\lambda}: \lambda \in \operatorname{Sp}(a, A)\} .
$$

Remark 1.2.11 Let $A$ be a real commutative Banach algebra with unit 1. Since the map ker: $\operatorname{Car}(A) \rightarrow M(A)$ is onto, we can consider the quotient topology on $M(A)$ : A subset $G \subset M(A)$ is defined to be open if $\operatorname{ker}^{-1}(G)$ is open in $\operatorname{Car}(A)$. Then it is easy to show that $M(A)$ is a compact Hausdorff space with respect to this topology. We shall call this topology the Gelfand topology in $M(A)$. The map ker is obviously continuous. It is also open because if $G$ is an open subset of $\operatorname{Car}(A)$, then $G \cup$ 
$\tau(G)$ is open. But $G \cup \tau(G)=\operatorname{ker}^{-1}(\operatorname{ker} G)$, so that $\operatorname{ker} G$ is open in $M(A)$. Essentially, $M(A)$ is the quotient space obtained from $\operatorname{Car}(A)$ by identifying $\phi$ with $\tau(\phi)$. Now let $a \in A$. Since $\hat{a}$ is a continuous complexvalued function on $\operatorname{Car}(A), \operatorname{Re} \hat{a}$ and $|\hat{a}|$ are real-valued continuous functions on $\operatorname{Car}(A)$. By Remark 1.2.7, the functions $\operatorname{Re} \hat{a}$ and $|\hat{a}|$ take constant values on $\operatorname{ker}^{-1}(M)$ for any $M$ in $M(A)$. Hence they induce the continuous maps $\operatorname{Re} \hat{a}: M(A) \rightarrow$ 同 and $|\hat{a}|: M(A) \rightarrow$ 同 defined by $\operatorname{Re} \hat{a}(M)$ $=\operatorname{Re} \phi(a)$ and $|\hat{a}|(M)=|\phi(a)|$, where $M \in M(A)$ and $\operatorname{ker} \phi=M$.

Let $\operatorname{Re} \hat{A}:=\{\operatorname{Re} \hat{a}: a \in A\}$ and $|\hat{A}|:=\{|\hat{a}|: a \in A\}$. It was shown in Limaye (1976) that the weak $\operatorname{Re} \hat{A}$ topology on $M(A)$ [i.e., the smallest topology on $M(A)$ making $\operatorname{Re} \hat{a}$ continuous for all $a$ in $A$ ] is the same as the weak $|\hat{A}|$ topology on $M(A)$. In fact, this topology is the same as the quotient topology defined above. To see this, consider a net $\left\{M_{\alpha}\right\}$ and an element $M$ in $M(A)$. Let $\phi_{\alpha}, \phi$ be in $\operatorname{Car}(A)$ such that $\operatorname{ker} \phi_{\alpha}=M_{\alpha}$ for each $\alpha$ and ker $\phi=M$. Now if $G$ is any neighborhood of $M$, then $\operatorname{ker}^{-1}(G)$ contains $\phi$ as well as $\phi$. Thus it is easy to check that $\left\{M_{\alpha}\right\}$ converges to $M$ in the quotient topology if and only if

(i) $\min \left\{\left|\hat{a}\left(\phi_{\alpha}\right)-\hat{a}(\phi)\right|,\left|\hat{a}\left(\phi_{\alpha}\right)-\hat{a}(\bar{\phi})\right|\right\} \rightarrow 0$ for every $a$ in $A$.

Similarly, $M_{\alpha}$ converges to $M$ in the weak $\operatorname{Re} \hat{A}$ (respectively, $|\hat{A}|$ ) topology if and only if:

(ii) $\operatorname{Re} \hat{a}\left(\phi_{\alpha}\right) \rightarrow \operatorname{Re} \hat{a}(\phi)$ for every $a$ in $A$ [respectively, (iii) $|\hat{a}|\left(\phi_{\alpha}\right) \rightarrow|\hat{a}|(\phi)$ for every $a$ in $\left.A\right]$.

We now show that statements (i), (ii), and (iii) are equivalent for every net $\left\{\phi_{\alpha}\right\}$ in $\operatorname{Car}(A)$. This implies that all the three topologies on $M(A)$ are the same. (i) obviously implies (ii) and (iii). "(ii) implies (iii)" follows from the relation

$$
|\hat{a}|^{2}=2(\operatorname{Re} \hat{a})^{2}-\operatorname{Re}\left(a^{2}\right)^{\wedge} \quad \text { for every } a \text { in } A .
$$

Similarly, "(iii) implies (ii)" follows by noting that

$$
\operatorname{Re} \hat{a}=\log \left|(\exp (a))^{\wedge}\right| \quad \text { for every } a \text { in } A .
$$

Thus (ii) and (iii) are equivalent. We shall now show that (ii) and (iii) imply (i). Let $a \in A, \hat{a}\left(\phi_{\alpha}\right)=x_{\alpha}+i y_{\alpha}$, and $\hat{a}(\phi)=x+i y$. (ii) and (iii) imply that $x_{\alpha} \rightarrow x$ and $x_{\alpha}^{2}+y_{\alpha}^{2} \rightarrow x^{2}+y^{2}$. Hence $y_{\alpha}^{2} \rightarrow y^{2}$; that is, $\left|y_{\alpha}\right| \rightarrow$ $|y|$. Now

$$
\begin{aligned}
& \min \left\{\left|\hat{a}\left(\phi_{\alpha}\right)-\hat{a}(\phi)\right|^{2}, \quad\left|\hat{a}\left(\phi_{\alpha}\right)-\hat{a}(\bar{\phi})\right|^{2}\right\} \\
& \quad=\min \left\{\left(x_{\alpha}-x\right)^{2}+\left(y_{\alpha}-y\right)^{2}, \quad\left(x_{\alpha}-x\right)^{2}+\left(y_{\alpha}+y\right)^{2}\right\} \\
& \quad=\left(x_{\alpha}-x\right)^{2}+\min \left\{\left(y_{\alpha}-y\right)^{2}, \quad\left(y_{\alpha}+y\right)^{2}\right\} \\
& \quad=\left(x_{\alpha}-x\right)^{2}+\left(\left|y_{\alpha}\right|-|y|\right)^{2} \rightarrow 0 .
\end{aligned}
$$


Corollary 1.2.12 Let $A$ be a real or complex commutative Banach algebra with unit 1 . Then the following statements are equivalent.

(i) $\|a\|^{2}=\left\|a^{2}\right\|$ for all $a$ in $A$.

(ii) $r(a)=\|a\|$ for all $a$ in $A$.

(iii) The Gelfand mapping $a \mapsto \hat{a}$ is an isometric isomorphism from $A$ onto a closed subalgebra of $C(\operatorname{Car}(A))$.

Proof.

(i) implies (ii): By induction, $\left\|a^{2^{m}}\right\|=\|a\|^{2^{m}}$ for $m=1,2, \ldots$ Hence, by Lemma 1.1.13,

$$
\begin{aligned}
r(a) & =\lim _{n \rightarrow \infty}\left\|a^{n}\right\|^{1 / n} \\
& =\lim _{m \rightarrow \infty}\left\|a^{2^{m}}\right\|^{1 / 2^{m}} \\
& =\|a\| .
\end{aligned}
$$

(ii) implies (iii): By Theorem 1.2.9 and Remark 1.2.10, $\hat{A}:=\{\hat{a}: a \in$ $A\}$ is a subalgebra, the map $a \mapsto \hat{a}$ is a homomorphism, and $\|\hat{a}\|=r(a)=$ $\|a\|$. Since $A$ is Banach, $A$ is closed in $C(\operatorname{Car}(A))$.

(iii) implies (i):

$$
\begin{aligned}
\left\|a^{2}\right\|=\left\|\left(a^{2}\right)^{\hat{y}}\right\| & =\left\|(\hat{a})^{2}\right\| \\
& =\|\hat{a}\|^{2} \quad \text { by definition of }\|\hat{a}\| \\
& =\|a\|^{2} .
\end{aligned}
$$

Every complex Banach algebra is also a real Banach algebra. To distinguish between complex Banach algebras and those real Banach algebras that behave in a different manner, Ingelstam (1964) introduced and studied some "reality conditions." These conditions indicate the extent to which a real Banach algebra is different from complex algebras. The strongest among these conditions was called "strict reality."

A real Banach algebra $A$ with unit 1 is called strictly real if $1+a^{2}$ is invertible for all $a$ in $A$. It is easy to see that if $A$ is strictly real, then $\operatorname{Sp}(a)$ $\subset$ I for all $a$ in $A$. In fact, if $a \in A$ and $s+i t \in \operatorname{Sp}(a)$ with $t \neq 0$, then $(a-s)^{2}+t^{2}$ is singular, that is, $1+((a-s) / t)^{2}$ is singular, in contradiction to the strict reality of $A$.

Now suppose that $A$ is commutative and strictly real. Then Theorem 1.2.9(vi) implies that the Gelfand transform $\hat{a}$ is real for each $a$ in $A$. Thus $\phi=\bar{\phi}$ for all $\phi$ in $\operatorname{Car}(A)$. As $\hat{A}$ is a real subalgebra of $C_{\mathbb{B}}(\operatorname{Car}(A))$ that contains the constant function $\hat{1}$ and separates the points of $\operatorname{Car}(A), \hat{A}$ is dense in $C_{\bar{\beta}}(\operatorname{Car}(A))$ by the real Stone-Weierstrass theorem. (See also Corollary 2.1.4 and the comments following it.) 
If $A$ is real Banach algebra with 1 satisfying $\|a\|^{2} \leq k\left\|a^{2}+b^{2}\right\|$ for all $a, b$ in $A$ with $a b=b a$ and some positive constant $k$, then $A$ is commutative by Theorem 1.1.30. It can also be proved that $\operatorname{Sp}(a) \subset$ R for each $a$ in $A$ [see Kulkarni and Limaye (1980, Theorem 2.4)]. Hence $\hat{A}$ is dense in $C_{\mathbb{R}}(\operatorname{Car}(A))$. Next, by Lemma 1.1.29(ii) and Theorem 1.2.9(vii), $\|a\| \leq$ $k r(a)=k\|\hat{a}\|$ for each $a$ in $A$. Hence $\hat{A}$ is uniformly closed; that is, $\hat{A}=C_{\mathbb{R}}(\operatorname{Car}(A))$. Thus $A$ is isomorphic and homeomorphic to $C_{\mathbb{R}}(X)$ for some compact Hausdorff space. This is a well-known theorem of Arens (Arens and Kaplansky, 1948).

\subsection{REAL FUNCTION ALGEBRAS}

We begin this section with the study of a few properties of the complex Banach algebra $C(X)$, described in Example 1.1.2. Recall that this is the algebra of all complex-valued continuous functions on a compact Hausdorff space, with pointwise operations and the supremum norm. Frequently, we shall need to take the supremum over subsets of $X$.

Notation For a nonempty subset $K$ of $X$ and $f$ in $C(X)$, we denote

$$
\|f\|_{K}:=\sup \{|f(x)|: x \in K\} .
$$

The subscript $K$ will be omitted when $K=X$.

Definition 1.3.1 Let $X$ be a compact Hausdorff space and $A$ be a nonempty subset of $C(X)$. For each $x$ in $X$, the evaluation map at $x$, denoted by $e_{x}$, is defined by

$$
e_{x}(f)=f(x) \quad \text { for } f \in A .
$$

It is easily seen that if $A$ is a subspace, then $e_{x}: A \rightarrow \mathbb{C}$ is a linear map, and if $A$ is a subalgebra, then $e_{x}$ is a homomorphism. If $A$ contains 1 , then $e_{x}(1)=1$ and hence $e_{x} \neq 0$. The next theorem shows that all the nonzero homomorphisms of $C(X)$ to $\mathbb{C}$ are of this form.

Theorem 1.3.2 Let $X$ be a compact Hausdorff space. Then $\operatorname{Car}(C(X))$ $=\left\{e_{x}: x \in X\right\}$.

Proof. We have already observed that $e_{x} \in \operatorname{Car}(C(X))$ for each $x$ in $X$. Now let $\phi \in \operatorname{Car}((C(X))$. By Remark 1.2.8, it is enough to prove that

$$
\text { ker } \phi=\operatorname{ker} e_{x} \quad \text { for some } x \text { in } X .
$$

For $f$ in $C(X)$, let $Z(f):=\{y \in X: f(y)=0\}$. We claim that

$$
K:=\cap\{Z(f): f \in \operatorname{ker} \phi\} \text { is nonempty. }
$$


Since each $Z(f)$ is a closed subset, by the finite intersection property of the compact space $X$, it is sufficient to prove that for $f_{1}, \ldots, f_{n}$ in $\operatorname{ker} \phi$, $Z\left(f_{1}\right) \cap \cdots \cap Z\left(f_{n}\right)$ is nonempty.

If possible, let $Z\left(f_{1}\right) \cap \cdots \cap Z\left(f_{n}\right)$ be empty. Then $g:=\sum_{i=1}^{n} f_{i} \bar{f}_{i} \in$ ker $\phi$. But since $f_{i}$ 's have no common zero, $g(y)>0$ for all $y$ in $X$. Hence $g$ is invertible, so that $\phi(g) \neq 0$. This contradiction proves the claim.

Now $I:=\{f \in C(X): f \equiv 0$ on $K\}$ is a proper ideal of $C(X)$. By the claim above, $\operatorname{ker} \phi \subset I$. Since $\operatorname{ker} \phi$ is a maximal ideal, we have $\operatorname{ker} \phi=$ $I$ and $K$ is a singleton, say $K=\{x\}$.

Corollary 1.3.3 Let $(C(X))_{R}$ denote $C(X)$, regarded as a real algebra. Then $\operatorname{Car}\left((C(X))_{R}\right)=\left\{e_{x}: x \in X\right\} \cup\left\{\bar{e}_{x}: x \in X\right\}$.

Proof. Follows from Theorem 1.3.2 and Remark 1.2.8.

Definition 1.3.4 (Involutions) Let $X$ be a topological space. A map $\tau: X \rightarrow X$ is called a topological involution on $X$ if $\tau$ is a homeomorphism and $\tau(\tau(x)$ ) $=x$ for all $x$ in $X$. (Note that, a priori, we could merely assume that $\tau$ is continuous.)

Let $A$ be a real or complex vector space. A linear involution on $A$ is a map $a \mapsto a^{*}$ of $A$ into $A$ satisfying the following axioms: For $a, b$ in $A$ and a scalar $\alpha$ :

(i) $(a+b)^{*}=a^{*}+b^{*}$.

(ii) $(\alpha a)^{*}=\bar{\alpha} a^{*}$.

(iii) $\left(a^{*}\right)^{*}=a$.

The scalar $\alpha$ can be a real or complex number depending on whether $A$ is a real or complex vector space. Note that if $A$ is a complex vector space, then a linear involution on $A$ is not a linear map; but it is conjugate linear.

Now let $A$ be an algebra over or $\mathbb{C}$. An algebra involution on $A$ is a linear involution on $A$ satisfying $(a b)^{*}=b^{*} a^{*}$ for all $a, b$ in $A$.

The adjective "topological," "linear," or "algebra" will be omitted when the type of an involution is clear from the context.

Let $X$ be a compact Hausdorff space and $\tau$ a topological involution on $X$. We now take a closer look at the algebra $C(X, \tau)$ defined in Example 1.1.2(b). Recall that

$$
C(X, \tau):=\{f \in C(X): f(\tau(x))=\bar{f}(x) \text { for all } x \in X\} .
$$

This algebra was defined explicitly in Kulkarni and Limaye (1981b). However, a careful reading of the papers of Arens and Kaplansky (1948) 
and Ingelstam (1964) will reveal the mention of this algebra in their papers. The main object of the present monograph is to investigate $C(X, \tau)$ and some of its subalgebras. One may ask: "Why should we study $C(X, \tau)$ ?"

The algebras $C(X)$ and $C_{R}(X)$ are well known in the literature. Many of their properties have been thoroughly investigated. We have already observed that if $\tau$ is the identity map on $X$, then $C(X, \tau)$ reduces to $C_{R}(X)$. On the other hand, $C(X)$ can also be treated as $C(Y, \tau)$, where $Y$ is a disjoint union of two copies of $X$ and $\tau$ is a homeomorphism that sends a point in one copy of $X$ to the corresponding point in the other copy. [Precisely, $Y=X \times\{0,1\}$ and $\tau((x, j))=(x,(j+1)$ modulo 2$)$ for $(x, j) \in Y$.] Identifying $X$ with one of the copies (say, $X \times\{0\}$ ), $X$ can be treated as a subspace of $Y$. Then every $f$ in $C(X)$ can be extended uniquely to $Y$ by requiring that the extension belongs to $C(Y, \tau)$.

Thus $C(X, \tau)$ is a more general object than $C_{R}(X)$ and $C(X)$. Hence every result that we prove about $C(X, \tau)$ will be valid for $C_{R}(X)$ and $C(X)$. On the other hand, it should be of interest to know how many properties of these better known algebras are shared by the algebra $C(X, \tau)$.

Theorem 1.3.5 Let $X$ be a compact Hausdorff space and $\tau$ a topological involution on $X$. Define $\sigma: C(X) \rightarrow C(X)$ by $\sigma(f)(x)=\bar{f}(\tau(x))$ for $f \epsilon$ $C(X), x \in X$. [In short, $\sigma(f)=\tilde{f} \circ \tau$.] Then:

(i) $\sigma$ is an algebra involution on $C(X)$ and

$$
C(X, \tau)=\{f \in C(X): \sigma(f)=f\} .
$$

(ii) $C(X)=C(X, \tau) \oplus i C(X, \tau)$; that is, every $h$ in $C(X)$ can be expressed uniquely as $f+i g$ with $f, g$ in $C(X, \tau)$.

(iii) $\sigma$ is an isometry.

(iv) For $f$ in $C(X)$, define $P(f)=[f+\sigma(f)] / 2$. Then $P$ is a continuous linear projection; that is, $P$ is a continuous linear map satisfying $P^{2}=P$ of $(C(X))_{R}$ onto $C(X, \tau)$.

(v) Every algebra involution on $C(X)$ arises from a topological involution on $X$ in the manner described above.

Proof.

(i) Obvious.

(ii) Since $\sigma$ is an algebra involution, for $f$ in $C(X), \sigma(f+\sigma(f))=$ $f+\sigma(f)$ and $\sigma((f-\sigma(f)) / i)=(f-\sigma(f)) / i$. Thus $f=(f+\sigma(f)) /$ $2+i((f-\sigma(f)) / 2 i)$ with $(f+\sigma(f)) / 2,(f-\sigma(f)) / 2 i$ in $C(X, \tau)$. Further, if $h=f+i g$ with $f$ and $g$ in $C(X, \tau)$, then $\sigma(h)=f-i g$ and hence $f=(h+\sigma(h)) / 2$ and $g=(h-\sigma(h)) / 2 i$. This proves the uniqueness of $f$ and $g$.

(iii) Let $f \in C(X)$. Then 


$$
\begin{aligned}
\|\sigma(f)\| & =\sup \{|\bar{f}(\tau(x))|: x \in X\} \\
& =\sup \{|f(y)|: y \in X\}, \quad \text { because } \tau \text { is an involution on } X \\
& =\|f\| .
\end{aligned}
$$

(iv) follows from (i) and (iii).

(v) Let $\sigma$ be an algebra involution on $C(X)$. We prove that $\sigma$ is induced by a topological involution $\tau$ on $X$. Let $x \in X$. Define $\phi_{x}: C(X) \rightarrow \mathbb{C}$ by $\phi_{x}(f)=\overline{\sigma(f)}(x), f \in C(X)$. It is routine to check that $\phi_{x} \in \operatorname{Car}(C(X))$. Hence, by Theorem 1.3.2, $\phi_{x}=e_{y}$ for a unique $y$ in $X$. We define $y=$ $\tau(x)$. Thus we have $f(\tau(x))=\overline{\sigma(f)}(x)$ for all $f$ in $C(X), x$ in $X$. Replacing $x$ by $\tau(x), f(\tau(\tau(x))=\overline{\sigma(f)}(\tau(x))=\sigma(\overline{(\sigma(f))})(x)=f(x)$ for all $f$ in $C(X)$. Hence $\tau(\tau(x))=x$ for all $x$ in $X$. Thus it remains only to show that $\tau$ is continuous. First note that since $\overline{\sigma(f)}(x)=f(\tau(x))$ for all $f$ in $C(X), x$ in $X$, and $\tau \circ \tau$ is the identity map, we have $\|\sigma(f)\|=\|f\|$. Hence $\sigma$ is continuous. Now suppose that a net $\left\{x_{\alpha}\right\}$ converges to a point $x$ in $X$. Then for every $f$ in $C(X), f\left(x_{\alpha}\right) \rightarrow f(x)$. Since $\sigma$ is continuous, this implies that $\sigma(f)\left(x_{\alpha}\right) \rightarrow \sigma(f)(x)$. Hence $f\left(\tau\left(x_{\alpha}\right)\right)=\overline{\sigma(f)}\left(x_{\alpha}\right) \rightarrow \overline{\sigma(f)}(x)=f(\tau(x))$. This means that $\tau\left(x_{\alpha}\right) \rightarrow \tau(x)$; that is, $\tau$ is continuous on $X$.

Note that $\tau=$ the identity map on $X$ gives rise to $\sigma(f)=\bar{f}$ and in this case $C(X)=C_{\text {南 }}(X)+i C_{\text {网 }}(X)$.

In proving assertion (v) of Theorem 1.3.5, we have used the following properties of $C(X)$ :

(i) If for $x, y$ in $X, f(x)=f(y)$ for all $f$ in $C(X)$, then $x=y$.

(ii) If for a net $\left\{x_{\alpha}\right\}$ and a point $x$ in $X, f\left(x_{\alpha}\right) \rightarrow f(x)$ for all $f$ in $C(X)$, then $x_{\alpha} \rightarrow x$.

These facts follow from Urysohn's lemma. Because $X$ is a compact Hausdorff space, if $x, y \in X$ and $x \neq y$, we can find a continuous function $f: X \rightarrow[0,1]$ such that $f(x)=1$ and $f(y)=0$.

Definition 1.3.6 Let $A$ be a set of functions on a set $X$. We say that $A$ separates the points of $X$ if for $x, y$ in $X$ and $x \neq y$, there exists $f$ in $A$ such that $f(x) \neq f(y)$.

Let $\tau$ be an involution on $X$ and $E$ be a set of functions on $X$ such that $u \circ \tau=u$ for every $u$ in $E$. We say that $E$ separates the points of $X / \tau$ if for $x, y$ in $X$ and $x \neq y, \tau(x) \neq y$, there exists $u$ in $E$ such that $u(x) \neq u(y)$.

Lemma 1.3.7 Let $X$ be a compact Hausdorff space and $\tau$ a topological involution on $X$. Let $x, y \in X$ and $x \neq y$.

(i) If $y=\tau(x)$, then there exists $f$ in $C(X, \tau)$ such that $f(x)=i$ and $f(y)=$ $-i$. 
(ii) If $y \neq \tau(x)$, then there exists $f$ in $C(X, \tau)$ such that $f(x)=1$ and $f(y)=0$.

In particular, $C(X, \tau)$ separates the points of $X$. and $\operatorname{Re} C(X, \tau)$ separates the points of $X / \tau$.

Proof. As in Theorem 1.3.5, for $h$ in $C(X)$, define $\sigma(h)=\bar{h} \circ \tau$. Then, by the same theorem, $\sigma(h+\sigma(h))=h+\sigma(h)$ and $\sigma(h \sigma(h))=h \sigma(h)$. Hence $h+\sigma(h)$ and $h \sigma(h) \in C(X, \tau)$.

(i) We can find $h$ in $C(X)$ such that $h(x)=i$ and $h(y)=0$. Let $f=$ $h+\sigma(h)$. Then $f \in C(X, \tau), f(x)=i$, and $f(y)=-i$.

(ii) We can find $h$ in $C(X)$ such that $h(x)=h(\tau(x))=1$ and $h(y)=0$. Let $f=h \sigma(h)$. Then $f \in C(X, \tau), f(x)=1$, and $f(y)=0$.

We now give the definition of a mathematical structure that is our main concern in this book.

Definition 1.3.8 Let $X$ be a compact Hausdorff space and $\tau$ a topological involution on $X$. A real function algebra on $(X, \tau)$ is a real subalgebra $A$ of $C(X, \tau)$ such that:

(i) $A$ is uniformly closed.

(ii) $1 \in A$.

(iii) $A$ separates the points of $X$.

A complex function algebra on a compact Hausdorff space $X$ is a complex subalgebra of $C(X)$, satisfying conditions (i), (ii), and (iii).

Notice that if $B$ is a complex subalgebra of $C(X)$ containing 1 and separating the points of $X$, we can assert that for $x, y$ in $X$ and $x \neq y$, there exists $f$ in $B$ such that $f(x)=0$ and $f(y)=1$. This can be seen as follows: There exists $g$ in $B$ such that $a:=g(x) \neq g(y):=b$. Let $f:=(g-a) /$ $(b-a)$. Then $f \in B, f(x)=0$, and $f(y)=1$. However, we cannot make the assertion above if $B$ is only a real subalgebra of $C(X)$. In particular, if $\tau$ is a topological involution on $X$, and $B$ is a (real) subalgebra of $C(X, \tau)$, then for every $f \in B, f(x)=0$ implies that $f(\tau(x))=0$.

Lemma 1.3.9 Let $X$ and $\tau$ be as in Definition 1.3.8. Let $A$ be a subalgebra of $C(X, \tau)$ that separates the points of $X$ and contains 1 . Let $x, y \in X$ with $x \neq y$. Then:

(i) If $y=\tau(x)$, there exists $f$ in $A$ such that $f(x)=i$ and $f(y)=-i$.

(ii) If $y \neq \tau(x)$, there exists $f$ in $A$ such that $f(x)=1$ and $f(y)=0$.

In particular, $\operatorname{Re} A$ separates the points of $X / \tau$.

Proof. Since $A$ separates the points of $X$, there exists $f_{1}$ in $A$ such that $\alpha_{1}:=f_{1}(x) \neq f_{1}(y)=: \beta_{1}$. 
(i) Let $y=\tau(x)$ and $\alpha_{1}=a+i b$. Then $\beta_{1}=a-i b$, so that $b \neq 0$. Now $f:=\left(f_{1}-a\right) / b \in A, f(x)=i$, and $f(y)=-i$.

(ii) Let $y \neq \tau(x)$. Then there is $f_{2}$ in $A$ such that $\alpha_{2}:=f_{2}(\tau(x)) \neq f_{2}(y)$ $:=\beta_{2}$. Now consider

$$
f:=\left[1-\frac{\left(f_{1}-\alpha_{1}\right)\left(f_{2}-\alpha_{2}\right)}{\left(\beta_{1}-\alpha_{1}\right)\left(\beta_{2}-\alpha_{2}\right)}\right]\left[1-\frac{\left(f_{1}-\bar{\alpha}_{1}\right)\left(f_{2}-\bar{\alpha}_{2}\right)}{\left(\bar{\beta}_{1}-\bar{\alpha}_{1}\right)\left(\bar{\beta}_{2}-\bar{\alpha}_{2}\right)}\right] .
$$

Let $r:=\left(\beta_{1}-\alpha_{1}\right)\left(\beta_{2}-\alpha_{2}\right)$. Then

$$
\begin{aligned}
f= & 1-\frac{2}{|r|^{2}}\left[\operatorname{Re}\left(\bar{\alpha}_{1} \bar{\alpha}_{2} r\right)+\operatorname{Re}\left(\bar{\alpha}_{2} r\right) f_{1}+\operatorname{Re}\left(\bar{\alpha}_{1} r\right) f_{2}+\operatorname{Re}(r) f_{1} f_{2}\right] \\
& +\frac{1}{|r|^{2}}\left[f_{1}^{2}-2 \operatorname{Re}\left(\alpha_{1}\right) f_{1}+\left|\alpha_{1}\right|^{2}\right]\left[f_{2}^{2}-2 \operatorname{Re}\left(\alpha_{2}\right) f_{2}+\left|\alpha_{2}\right|^{2}\right] .
\end{aligned}
$$

Hence $f \in A$. Now it is easy to check that $f(x)=1$ and $f(y)=0$.

Like complex Banach algebras, complex function algebras have been objects of detailed investigation. Most books on Banach algebras contain some material devoted to complex function algebras. In addition, since the late 1960s several books dealing entirely with complex function algebras have appeared: for example, Browder (1969), Gamelin (1969), Stout (1971), and Suciu (1975). On the other hand, real function algebras are of recent origin. These algebras were defined for the first time in Kulkarni and Limaye (1981b). Since then, many mathematicians have attempted to develop the theory of real function algebras along the lines of complex function algebras. Our aim in this book is to present a systematic account of these attempts. We shall soon establish that the class of real function algebras includes that of complex function algebras. Hence every result about real function algebras can be regarded as a generalization of the corresponding result about complex function algebras.

Assume that condition (i) of Definition 1.3.8 is replaced by the following:

(i) $A$ is a Banach algebra with respect to some norm.

Then the resulting algebra is known as a real (or complex) Banach function algebra. Obviously, every (real or complex) function algebra is a Banach function algebra. The Wiener algebra (Example 1.1.5) is a complex Banach function algebra, which is not a function algebra, because the norm defined there is not the supremum norm.

We now discuss a few examples of function algebras other than the trivial ones: For a compact Hausdorff space $X, C(X)$ is a complex func- 
tion algebra, and if $\tau$ is a topological involution on $X$, then $C(X, \tau)$ is a real function algebra.

Example 1.3.11 Let $X$ be a compact subset of $\mathbb{C}$ and $B$ the algebra of all functions that are uniform limits of sequences of rational functions $p / q$, where $p$ and $q$ are polynomials and $q$ has no zero on $X$. Then $B$ is a $C(X)$ that are analytic in the open unit disk $\{z \in \mathbb{G}:|z|<1\}$. Then $B$ is a complex function algebra, known in the literature as the disk algebra. This algebra serves as a model for the development of the theory of complex function algebras. Now, define a map $\tau: X \rightarrow X$ by $\tau(z)=\bar{z}$ for $z \in X$. Let $A=\{f \in B: f(\tau(z))=\overline{f(z)}\}$. Then $A$ equals the set of all uniform limits of polynomials with real coefficients on $X . A$ is a real function algebra. In fact, $A=B \cap C(X, \tau)$. $A$ is called the real disk algebra and is useful as a model to develop the theory of real function algebras. We now prove that the carrier space of $A$ can be identified with the disk $X$, that is,

$$
\operatorname{Car}(A)=\left\{\mathbf{e}_{\lambda}: \lambda \in X\right\} .
$$

Clearly, if $\lambda \in X, e_{\lambda} \in \operatorname{Car}(A)$. To prove the reverse inclusion, consider $\phi \in \operatorname{Car}(A)$. Consider the polynomial $z$ in $A: z$ is the function defined as $z(\lambda)=\lambda$ for all $\lambda$ in $X$. Let $\phi(z)=\lambda$. Since $\|z\|=1$, we have $|\lambda| \leq 1$. Thus $\lambda \in X$. Since $\phi \in \operatorname{Car}(A), \phi\left(z^{n}\right)=\lambda^{n}$ for $n=1,2, \ldots$. Hence $\phi(p)=$ $p(\lambda)$ for every polynomial $p$ with real coefficients and hence $\phi(f)=f(\lambda)$ for every $f \in A$. Thus $\phi=e_{\lambda}$.

Similarly, we can prove that $\operatorname{Car}(B)=\left\{e_{\lambda}: \lambda \in X\right\}$. (Note that we have used the same symbol, $e_{\lambda}$, for a homomorphism from $B$ to $\mathbb{C}$.) Thus the maximal ideals of $B$ are of the form

$$
M_{z}=\{f \in B: f(z)=0\},
$$

where $z \in \mathbb{C}$ and $|z| \leq 1$.

The closed ideals of the disk algebra $B$ can be described as follows (Rudin, 1957). Let $J$ be a closed ideal in $B$. Then there is a bounded analytic function $q$ on the open unit disk and a closed subset $E$ of the unit circle such that the Lebesgue measure of $E$ is zero,

$$
\begin{aligned}
\left|\lim _{r \rightarrow 1} q\left(r e^{i \theta}\right)\right| & =1 \quad \text { for almost all } \theta, \\
J & =\{q f: f \in B, f=0 \text { on } E\} .
\end{aligned}
$$

More generally, we may consider the algebra $B$ consisting of uniform limits of sequences of polynomials on a compact subset of $\mathbb{C}$, and the algebra $A$ consisting of uniform limits of sequences of polynomials with 
real coefficients on a compact subset of $\mathbb{C}$ that is symmetric about the real axis (Definition 1.1.24).

Example 1.3.11 Let $X$ be a compact subset of $\mathbb{C}$ and $B$ the algebra of all functions that are uniform limits of sequences of rational functions $p$ / $q$, where $p$ and $q$ are polynomials and $q$ has no zero on $X$. Then $B$ is a complex function algebra. Further, if $X$ is symmetric about the real axis, we can define $\tau: X \rightarrow X$ by $\tau(z)=\bar{z}, z \in X$. Let $A:=\{f \in B: f(\tau(z))=$ $\bar{f}(z)$ for all $z$ in $X\}$. Then $A$ is a real function algebra consisting of all uniform limits of sequences of rational functions $p / q$, where $p$ and $q$ are polynomials with real coefficients and $q$ does not have any zero on $X$.

Example 1.3.12 Let $0<c<d$ and $X$ be the annular region $X:=\{z \in$ $\mathbb{C}: c \leq|z| \leq d\}$. Let

$$
B:=\{f \in C(X): f \text { is analytic in the interior of } X\} \text {. }
$$

Then $B$ is a complex function algebra on $X$. Now let $0<c<1$ and $d=$ $1 / c$. Then we can define a map $\tau: X \rightarrow X$ by $\tau(z)=-1 / \bar{z}$. This map is a topological involution on $X$. Define

$$
A:=\{f \in B: f(\tau(z))=\bar{f}(z) \text { for all } z \in X\} .
$$

Then $A$ is a real function algebra on $(X, \tau)$.

More generally, an algebra $B$ can similarly be defined on any compact Riemann surface with a nonempty boundary. [We refer the reader to Springer (1957) for an introduction to Riemann surfaces.] Now let $Y$ be a compact nonorientable Klein surface with nonempty boundary $\partial Y$. Then $Y$ admits an orienting double $(X, p, \tau)$, where $X$ is a compact Riemann surface with boundary $\partial X, p: X \rightarrow Y$ is a $2: 1$ covering map such that $p(\partial X)$ $=\partial Y, p^{-1}(\partial Y)=\partial X$, and $\tau: X \rightarrow X$ is an antianalytic involution of $X$ such that $p \circ \tau=p$. [For definitions and proof, see Alling and Greenleaf (1971, p. 140).] Then we can define algebras $B$ and $A$ as above. $A$ is called the standard algebra on a Klein surface $Y$. In the particular case mentioned above, the annular region $X$, with $d=1 / c$, is the orienting double of a Möbius strip. A description of the closed ideals of the function algebras $B$ and $A$ is given in Alling and Limaye (1972) on the lines of the description of the closed ideals of the disk algebra mentioned in Example 1.3.10.

It follows from the comments at the end of Section 1.2 that if a real function algebra $A$ on $(X, \tau)$ is strictly real, then $\tau$ is the identity map on $X$ and $A=C_{\text {( }}(X)$. Hwang (1990) has studied the implications of this and other reality conditions in the context of a real function algebra. 
Definition 1.3.13 A real uniform algebra is a real commutative Banach algebra $A$ with unit 1 such that $\|a\|^{2}=\left\|a^{2}\right\|$ for all $a$ in $A$.

Remark 1.3.14 One can similarly define a complex uniform algebra. In fact, in this case, the assumption of commutativity need not be made, a priori, as any complex Banach algebra $B$ satisfying $\|a\|^{2}=\left\|a^{2}\right\|$ for all $a$ $\in B$ is commutative by Theorem 1.1.30(i). Example 1.1.4 shows that this theorem does not hold for real Banach algebras.

Real uniform algebras were first defined in Limaye and Simha (1975). Obviously, every real function algebra is a real uniform algebra. Here are some further examples of real uniform algebras.

Example 1.3.15 Let $D:=\{z \in \mathbb{C}:|z|<1\}$ be the open unit disk and $A$ be the set of all complex-valued bounded analytic functions $f$ on $D$ satisfying $f(\bar{z})=\overline{f(z)}$ for all $z \in D$. With the supremum norm, $A$ is obviously a real uniform algebra.

Example 1.3.16 Let $\mu$ be a positive measure on a $\sigma$-algebra on a measure space $X$, and let $\tau: X \rightarrow X$ be a map such that $\tau(\tau(x))=x$ for all $x$ in $X$, and $\tau(E)$ is measurable whenever $E$ is a measurable subset of $X$. [As a particular case, we may consider $X=R, \mu$ the Lebesgue measure, and $\tau(x)=-x$ for $x$ in R.] Let

$$
A:=\left\{f \in L^{\infty}(\mu): f(\tau(x))=\vec{f}(x) \text { for all } x \text { in } X\right\} .
$$

$A$ is clearly a real uniform algebra.

We will now show that real uniform algebras are real function algebras in disguise. To penetrate this disguise, we have to take help of the Gelfand representation developed in Section 1.2.

Theorem 1.3.17 Every real uniform algebra is isometrically isomorphic to a real function algebra.

Proof. Let $A$ be a real uniform algebra. Let $X=\operatorname{Car}(A)$ and $\tau: X \rightarrow X$ be the map $\tau(\phi)=\bar{\phi}$. By Ingelstam's theorem (Theorem 1.2.9), $X$ is a compact Hausdorff space and $\tau$ is a topological involution on $X$. Let $\hat{A}=$ $\{\hat{a}: a \in A\}$, where $\hat{a}$ denotes the Gelfand transform of $a$. By Corollary 1.2.12, the Gelfand mapping $a \mapsto \hat{a}$ is an isometric isomorphism of $A$ onto $\hat{A}$. Thus it suffices to prove that $\hat{A}$ is a real function algebra on $(X, \tau)$. Clearly, for each $a$ in $A$ and $\phi$ in $X, \hat{a}(\tau(\phi))=\Phi(a)=\hat{a}(\phi)$. Hence $\hat{a} \in$ $C(X, \tau)$. Thus $\hat{A}$ is a real subalgebra of $C(X, \tau)$. It contains the constant 
function $\hat{1}$; it obviously separates the points of $X$. Since $\|\hat{a}\|=\|a\|$ for each $a$ in $A, \hat{A}$ is uniformly closed.

Note that we need not choose $X$ to be the entire carrier space of $A$ in proving Theorem 1.3.17. Instead, we could as well choose $Y$ to be any closed subset of $X$ satisfying $\tau(Y)=Y$ and

$$
\sup \{|\phi(a)|: \phi \in X\}=\sup \{|\phi(a)|: \phi \in Y\}
$$

for every $a$ in $A$. Then $\hat{A}_{\mid Y}:=\left\{\left.\hat{a}\right|_{Y}: a \in A\right\}$ is a real function algebra on $\left(Y,\left.\tau\right|_{Y}\right)$, which is isometrically isomorphic to $A$. A natural choice for such a set $Y$ can often be made if we know something more about the algebra $A$. This happens for a class of real uniform algebras studied by Mehta and Vasavada (1986).

Corollary 1.3.18 Let $X$ be a compact Hausdorff space and $A$ be a uniformly closed real subalgebra of $C(X)$ containing constants and separating the points of $X$. For $\phi$ in $\operatorname{Car}(A)$, let $\tau(\phi)=\bar{\phi}$. Identifying $x \in X$ with $e_{x} \in \operatorname{Car}(A)$, let $Y=X \cup \tau(X) \subset \operatorname{Car}(A)$. Then $\hat{A}_{\mid Y}$ is a real function algebra on $\left(Y, \tau_{\mid Y}\right)$, and $A$ is isometrically isomorphic to $\hat{A}_{\mid Y}$.

Proof. Every $\phi$ in $Y$ equals $e_{x}$ or $\bar{e}_{x}$ for some $x$ in $X$, so that for every $f$ in $A, \hat{f}(\phi)=\hat{f}(\tau(\phi))$, and

$$
\left\|\hat{f}_{\mid Y}\right\|=\sup \{|\hat{f}(\phi)|: \phi \in Y\}=\sup \{|f(x)|: x \in X\}=\|f\| .
$$

Hence $\hat{A}_{\mid Y}$ is uniformly closed in $C\left(Y, \tau_{\mid Y}\right)$. Clearly, $\hat{1}_{Y Y}$ is a constant function on $Y$ and $\hat{A}_{\mid Y}$ separates the points of $Y$. Thus $\hat{A}_{\mid Y}$ is a real function algebra on $\left(Y, \tau_{\mid Y}\right)$. It is also obvious that the map $f \mapsto \hat{f}_{\mid Y}$ is an isometric isomorphism of $A$ onto $\hat{A}_{\mid Y}$.

\section{Remark 1.3.19}

(i) In Corollary 1.3.18, suppose that $X$ and $A$ have the following property: For each $x$ in $X$, there is $y$ in $X$ such that $f(y)=\bar{f}(x)$ for all $f$ in $A$. This means that $e_{y}=\bar{e}_{x}$; that is, $\tau(x) \in X$. Hence $Y=X$ and $\tau$ is an involution on $X$.

As an example of this case, we may consider

$$
A:=\{f \in C([0,1]): f(1-t)=\bar{f}(t) \text { for } 0 \leq t \leq 1\} .
$$

(ii) At the other extreme, suppose that $X$ and $A$ have the following property: For every $x, y$ in $X, \bar{e}_{x} \neq e_{y}$. Thus $\tau(x) \notin X$ for each $x$ in $X$. Hence $Y$ is the disjoint union of $X$ and $\tau(X)$.

As an example of this case, we may consider $A=C([0,1])$. We may note that if $A$ is any complex function algebra, then $A$ has property (ii), 
because if $x \neq y$, we can find $f$ in $A$ with $f(x)=0, f(y)=1$, and if $x=y$, we can take $f$ as the constant function $i$.

Thus every complex function algebra on $X$ can be regarded as a real function algebra on $(Y, \tau)$, where $Y$ is a disjoint union of two copies of $X$ and the involution $\tau$ sends a point in one copy of $X$ to the corresponding point in the other copy. This justifies our earlier observation that the class of real function algebras includes that of complex function algebras.

Note that if $A$ is a complex function algebra on a compact Hausdorff space $X$, then it is not possible to define any topological involution $\tau^{\prime}$ on $X$ in such a way that $A$ becomes a real function algebra on $\left(X, \tau^{\prime}\right)$, because the constant function $i$ does not belong to $C\left(X, \tau^{\prime}\right)$ for any $\tau^{\prime}$.

(iii) In between the two extremes mentioned above, we may consider the following property: For every $x, y$ in $X$ with $x \neq y$, there is $f$ in $A$ such that $f(x) \neq \tilde{f}(y)$. This happens, in particular, if $\operatorname{Re} A$ separates the points of $X$. Let

$$
Z:=\{x \in X: f(x) \text { is a real for all } f \text { in } A\} .
$$

Then $\tau(x)=x$ for all $x$ in $Z$ and $\tau(x) \in Y \backslash X$ for all $x$ in $X \backslash Z$. Thus $Y$ is a disjoint union of $X$ and $\tau(X \backslash Z)$. As an example of this case, we may consider

$$
A:=\left\{f \in C([0,1]): f\left(\frac{1}{2}\right) \text { is real }\right\} .
$$

Given a real function algebra $A$, we will now show that there is a complex function algebra that can be viewed as a complexification of $A$ (see Definition 1.1.6).

Theorem 1.3.20 Let $X$ be a compact Hausdorff space and $\tau$ a topological involution on $X$. Let $\sigma(f)=\tilde{f} \circ \tau$ be the algebra involution induced by $\tau$ on $C(X)$. Let $A$ be a real subspace of $C(X, \tau)$ and define

$$
B:=\{f+i g: f, g \in A\} .
$$

Then

(i) $\sigma(B)=B$ and $A=\{h \in B: \sigma(h)=h\}=B \cap C(X, \tau)$.

(ii) For $f, g$ in $C(X, \tau)$,

$$
\max \{\|f\|,\|g\|\} \leq\|f+i g\| \leq\|f\|+\|g\| .
$$

(iii) $B$ is uniformly closed (respectively, separates the points of $X$, contains 1 , is an algebra) if and only if $A$ is uniformly closed (respectively, separates the points of $X$, contains 1 , is an algebra).

(iv) Let $A$ be a real function algebra on $(X, \tau)$. For $\phi$ in $\operatorname{Car}(A)$, define

$$
\alpha(\phi)(f+i g):=\phi(f)+i \phi(g)
$$


for $f, g$ in $A$. Then $\alpha(\phi) \in \operatorname{Car}(B)$. The map $\alpha$ is a bijection between $\operatorname{Car}(A)$ and $\operatorname{Car}(B)$.

Proof. We shall use the properties of $\sigma$ proved in Theorem 1.3.5.

(i) Let $h=f+i g \in B, f, g \in A$. Since $\sigma$ is an algebra involution, $\sigma(h)$ $=\sigma(f+i g)=f-i g \in B$. Also, if $\sigma(h)=h$, then $h=f \in A$. This proves (i).

(ii) Let $f, g \in C(X, \tau)$. Since $\sigma$ is an isometry, $\|f+i g\|=\|\sigma(f+i g)\|$ $=\|f-i g\|$. Hence $\|f\| \leq\left(\frac{1}{2}\right)(\|f+i g\|+\|f-i g\|)=\|f+i g\|$. Similarly, $\|g\| \leq\|f+i g\|$. This proves (ii).

(iii) The "only if" parts are trivial. Let now $A$ be uniformly closed. Let $f_{n}, g_{n} \in A$ and $f_{n}+i g_{n}$ be a sequence in $B$, converging to a function $h \in$ $C(X)$. Then since $\sigma$ is an isometry, $f_{n}-i g_{n}=\sigma\left(f_{n}+i g_{n}\right) \rightarrow \sigma(h)$ as $n \rightarrow$ $\infty$. Hence $f_{n} \rightarrow(h+\sigma(h)) / 2$ and $g_{n} \rightarrow(h-\sigma(h)) / 2 i$ as $n \rightarrow \infty$. Since $A$ is uniformly closed, $(h+\sigma(h)) / 2$ and $(h-\sigma(h)) / 2 i$ are in $A$. Hence $h=(h$ $+\sigma(h)) / 2+i(h-\sigma(h)) / 2 i \in B$. This proves that $B$ is uniformly closed.

Since $A \subset B$, if $A$ contains 1 , so does $B$, and if $A$ separates the points of $X$, so does $B$. It is easy to see that if $A$ is an algebra, then $B$ is an algebra.

(iv) Let $\phi \in \operatorname{Car}(A)$. It is straightforward to check that $\alpha(\phi) \in \operatorname{Car}(B)$, $\left.\alpha(\phi)\right|_{A}=\phi$, and for any $\psi \in \operatorname{Car}(B),\left.\psi\right|_{A} \in \operatorname{Car}(A)$ with $\alpha\left(\left.\psi\right|_{A}\right)=\psi$. This proves (iv).

In view of property (ii) of Theorem 1.3.20, every $h$ in $B$ can be written uniquely as $h=f+i g$ for some $f, g$ in $A$. Hence we can regard $B$ as the complexification of $A$ (Definition 1.1.6) whenever $A$ is a real algebra. The supremum norm on $B$ satisfies the properties of the norm $p$ mentioned after Definition 1.1.6.

We may note that if $X$ is a compact Hausdorff space and $\tau$ a topological involution on $X$, then $C(X)$ is the complexification of $C(X, \tau)$. In particular, $C(X)$ is the complexification of $C_{\text {P }}(X)$. The disk algebra of Example 1.3.10 is the complexification of the real disk algebra. Further, the algebra $B$ in Example 1.3.11 is the complexification of the algebra $A$ in the same example. The same thing can be said about the algebras $B$ and $A$ of Example 1.3.12.

Corollary 1.3.21 Let $X$ be a compact Hausdorff space and $\tau$ be a topological involution on $X$. Then

$$
\operatorname{Car}(C(X, \tau))=\left\{e_{x}: x \in X\right\} .
$$

Proof. Clearly, $e_{x} \in \operatorname{Car}(C(X, \tau))$ for each $x$ in $X$. Now, let $\phi \in$ $\operatorname{Car}(C(X, \tau))$. Then, by Theorem 1.3.20, $\alpha(\phi) \in \operatorname{Car}(C(X))$. Hence, by Theorem 1.3.2, $\alpha(\phi)=e_{x}$ for some $x$ in $X$. Hence $\phi=e_{x}$ on $C(X, \tau)$. 
One can also give a direct proof of Corollary 1.3.21, which is similar to the proof of Theorem 1.3.2.

In some problems, we need to know when a given complex function algebra can be viewed as a complexification of a real function algebra. The following theorem gives a criterion.

Theorem 1.3.22 Let $X$ be a compact Hausdorff space, $\tau$ a topological involution on $X, \sigma$ the algebra involution on $C(X)$ given by $\sigma(f)=\tilde{f} \circ \tau$, $f \in C(X)$, and $B$ a complex function algebra on $X$. If $\sigma(B)=B$, then $B$ can be regarded as a complexification of a real function algebra $A$ on $(X, \tau)$ given by $A:=\{h \in B: \sigma(h)=h\}$.

Proof. Let $h \in B$. Since $\sigma(B)=B$, we have $f:=(h+\sigma(h)) / 2$ and $g:=$ $(h-\sigma(h)) / 2 i$ belong to $B$. Further, since $\sigma(f)=f$ and $\sigma(g)=g, f, g \in A$. Also, $h=f+i g$. Using properties of $\sigma$, it is easy to prove that if $h=f_{1}$ $+i g_{1}$, with $f_{1}, g_{1}$ in $A$, then $f=f_{1}$ and $g=g_{1}$. Thus every element $h$ of $B$ can be uniquely expressed as $h=f+i g$ with $f, g$ in $A$. Now it remains only to show that $A$ is a real function algebra on $(X, \tau)$. It is straightforward to check that $A$ is a real subalgebra of $C(X, \tau)$. Since $\sigma$ is an isometry and $A$ is the set of all fixed points of $\sigma, A$ is uniformly closed. Also, $\sigma(1)$ $=1 \in A$. Now let $x, y \in X$ and $x \neq y$. Since $B$ separates the points of $X$, there exists $h$ in $B$ such that $h(x) \neq h(y)$. Now $h=f+i g$ for some $f, g \in$ $A$. Hence $f(x) \neq f(y)$ or $g(x) \neq g(y)$. Thus $A$ separates the points of $X$.

Theorem 1.3.20 allows us to use the complexification technique mentioned after Definition 1.1.6 to deduce results for real function algebras from the corresponding results for complex function algebras. We shall, however, prefer to develop the theory of real function algebras intrinsically. Only when this seems infeasible shall we resort to the complexification technique, with a hope that someone will furnish intrinsic proofs.

Let $X$ be a compact Hausdorff space, $\tau$ a topological involution on $X$, and $A$ a real function algebra on $(X, \tau)$. The most natural question that one can ask about $A$ is: When is $A$ equal to the whole of $C(X, \tau)$ ? This question is the central theme of Chapter 2.

\subsection{DEFORMATIONS OF A REAL FUNCTION ALGEBRA}

In this section we study the effect of introducing a small change in the operation of multiplication in a real function algebra. This subject is yet to be developed to its full potential even in the case of complex function 
algebras. Our treatment is, therefore, necessarily cursory. The material in this section is not used subsequently in this book.

We first make precise the meaning of the terminology "small change." There are two essentially different ways of doing this.

Definition 1.4.1 Let $A$ be a real (respectively, complex) Banach algebra and let the original multiplication in $A$ be denoted by juxtaposition as usual. Let $\varepsilon>0$. A binary operation $*$ defined on $A$ is called an algebraic $\varepsilon$-perturbation of $A$ if $(A, *)$ is a real (respectively, complex) algebra and

$$
\|a * b-a b\| \leq \varepsilon\|a\|\|b\| \quad \text { for all } a, b \text { in } A .
$$

The algebra $(A, *)$ is called an algebraic $\varepsilon$-deformation of $A$.

$A$ is called stable (or rigid) if there exists $\varepsilon_{0}>0$ such that every algebraic $\varepsilon$-deformation of $A$ is isomorphic to $A$ for $0 \leq \varepsilon \leq \varepsilon_{0}$.

Definition 1.4.1 implies that the original multiplication and the new multiplication are close to each other as bilinear maps on $A$. Note that if $*$ is any continuous multiplication on $A$, then there exists $k>0$ such that $\| a$ $* b\|\leq k\| a\|\| b \|$ for all $a, b$ in $A$ and hence

$$
\|a * b-a b\| \leq(k+1)\|a\|\|b\| \quad \text { for all } a, b \text { in } A .
$$

Thus $*$ is an algebraic $(k+1)$-perturbation of $A$. We are, therefore, interested in $\varepsilon$-perturbations of $A$ for small values of $\varepsilon$, particularly $\varepsilon<1$ in many cases.

Perturbations of complex Banach algebras have been studied in recent years by Kadison and Kastler (1972), Philips (1973), Johnson (1977), and Raeburn and Taylor (1979). The investigations concerning the perturbations of complex function algebras were initiated by Rochberg (1979) and continued in several articles by Rochberg and Jarosz. The lecture notes by Jarosz (1985) contain a detailed account of all these results.

Definition 1.4.2 Let $A$ be a real (respectively, complex) Banach algebra. Let $\varepsilon>0$. A binary operation $*$ defined on $A$ is called a metric $\varepsilon$ perturbation of $A$ if there is a real (respectively, complex) Banach algebra $\left(A_{1}, \cdot\right)$ and a linear, continuous bijection $T: A \rightarrow A_{1}$ such that

$$
a * b=T^{-1}(T a \cdot T b)
$$

for all $a, b$ in $A$ and $\|T\|\left\|T^{-1}\right\| \leq 1+\varepsilon . A_{1}$ is then called a metric $\varepsilon$ deformation of $A$.

It is easy to see that $(A, *)$ is a real (respectively, complex) algebra.

A natural question at this stage is whether an algebraic (or metric) 
deformation of a function algebra is a function algebra. The next theorem deals with this question for an algebraic perturbation. Since every function algebra is a uniform algebra (Definition 1.3.13), and vice versa (Theorem 1.3.17), it is enough to establish the results for uniform algebras.

Since $\|a * b\| \leq(1+\varepsilon)\|a\|\|b\|$ for an $\varepsilon$-algebraic perturbation of $A$, the spectral radius of $a$ in $(A, *)$ can be defined as in 1.1.12. Also, 1.1.13 and the result following it can be proved.

Theorem 1.4.3 Let $A$ be a real or complex uniform algebra and $*$ be an algebraic $\varepsilon$-perturbation of $A$ with $0 \leq \varepsilon<1$. Let $N(f)$ denote the spectral radius of $f$ in $(A, *)$. Then:

(i) $(1-\varepsilon)\|f\| \leq N(f) \leq(1+\varepsilon)\|f\|$ for all $f$ in $A$.

(ii) If $*$ is commutative, then $(A, *, N)$ is a uniform algebra. Moreover, $*$ is a metric $\varepsilon_{1}$-perturbation of $A$, where $\varepsilon_{1}=2 \varepsilon /(1-\varepsilon)$.

Proof.

(i) Let $f \in A$. Then

$$
\|\| f * f\|-\| f^{2}\|\mid \leq\| f * f-f^{2}\|\leq \varepsilon\| f \|^{2} .
$$

Since $\left\|f^{2}\right\|=\|f\|^{2}$, we get

$$
(1-\varepsilon)\|f\|^{2} \leq\|f * f\| \leq(1+\varepsilon)\|f\|^{2} .
$$

By induction, we see that for all $n=1,2, \ldots$,

$$
(1-\varepsilon)^{2^{n}}(1-\varepsilon)^{2^{n+1}}\|f\|^{2^{n}} \leq \underbrace{\|f * \cdots * f\|}_{2^{n} \text { times }} \leq(1+\varepsilon)^{2^{n}-1}\|f\|^{2^{n}} .
$$

Taking the $2^{n}$ th root of each term in the inequality above, letting $n \rightarrow \infty$, and using the spectral radius formula (Theorem 1.1.19), we obtain

$$
(1-\varepsilon)\|f\| \leq N(f) \leq(1+\varepsilon)\|f\| .
$$

(ii) (i) implies that $f=0$ if and only if $N(f)=0$. If $*$ is commutative, it follows from (iv) and (vii) of Theorem 1.2.9 that the spectral radius $N$ is a norm on $(A, *)$. By Corollary 1.2.12, $N(f * f)=N(f)^{2}$ for all $f$ in $A$. Now we show that $*$ has a unit. Define a map $T_{1}: A \rightarrow A$ by $T_{1}(f)=1 * f$ for $f$ in $A$, and let $I$ denote the identity map on $A$. For $f$ in $A$,

$$
\|1 f-1 * f\| \leq \varepsilon\|f\|, \quad\|1 * f\| \leq(1+\varepsilon)\|f\| .
$$

Hence $T_{1}$ is a bounded linear map on $A$ and $\left\|I-T_{1}\right\|<1$, so that $T_{1}$ is invertible [Lemma 1.1.14(i)]. Let $e=T_{1}^{-1}(1)$. Then $1=T_{1}(e)=1 * e$. Now for all $f$ in $A, T_{1}(e * f)=1 *(e * f)=(1 * e) * f=1 * f=T_{1}(f)$. Since $T_{1}$ is injective, this implies that $e * f=f$ for all $f$ in $A$. Hence $e$ is a unit in $(A, *)$. Thus $(A, *)$ is a uniform algebra. 
Next consider the map $T: A \rightarrow(A, *)$ given by $T(f)=f$ for all $f$ in $A$. Then from (i), $\|T\| \leq 1+\varepsilon$ and $\left\|T^{-1}\right\| \leq 1 /(1-\varepsilon)$. Thus $\|T\|\left\|T^{-1}\right\| \leq(1$ $+\varepsilon) /(1-\varepsilon)$. Since $(1+\varepsilon) /(1-\varepsilon)=1+2 \varepsilon /(1-\varepsilon), T$ defines a metric $\varepsilon_{1}$-perturbation of $A$, where $\varepsilon_{1}=2 \varepsilon /(1-\varepsilon)$.

Notice that in (ii) of Theorem 1.4.3, we have assumed that $*$ is commutative. Jarosz (1985) showed that such an assumption is not necessary for complex uniform algebras. We shall now prove this result of Jarosz and use it along with the complexification technique to deduce a similar result for real function algebras. (See the comments at the end of Section 1.3.)

Lemma 1.4.4 Let $X$ be a compact Hausdorff space, $\tau$ a topological involution on $X$, and $A$ a real function algebra on $(X, \tau)$. Suppose that $*$ is an algebraic $\varepsilon$-perturbation of $A$. Let $B:=\{f+i g: f, g \in A\}$. Define a binary operation, also denoted by $*$, on $B$ as follows: For $h_{1}=f_{1}+i g_{1}, h_{2}=f_{2}+$ $i g_{2} \in B$ with $f_{1}, g_{1}, f_{2}, g_{2} \in A$, let

$$
h_{1} * h_{2}=f_{1} * f_{2}-g_{1} * g_{2}+i\left(f_{1} * g_{2}+g_{1} * f_{2}\right) \text {. }
$$

Then $*$ is an algebraic $4 \varepsilon$-perturbation of $B$.

Proof. For $h_{1}$ and $h_{2}$ as above, we have

$$
h_{1} h_{2}=f_{1} f_{2}-g_{1} g_{2}+i\left(f_{1} g_{2}+g_{1} f_{2}\right) \text {. }
$$

Hence

$$
\begin{aligned}
\left\|h_{1} * h_{2}-h_{1} h_{2}\right\| \leq & \left\|f_{1} * f_{2}-f_{1} f_{2}\right\|+\left\|g_{1} * g_{2}-g_{1} g_{2}\right\| \\
& +\left\|f_{1} * g_{2}-f_{1} g_{2}\right\|+\left\|g_{1} * f_{2}-g_{1} f_{2}\right\| \\
\leq & \varepsilon\left(\left\|f_{1}\right\|\left\|f_{2}\right\|+\left\|g_{1}\right\|\left\|g_{2}\right\|+\left\|f_{1}\right\|\left\|g_{2}\right\|+\left\|g_{1}\right\|\left\|f_{2}\right\|\right) \\
\leq & 4 \varepsilon\left\|h_{1}\right\|\left\|h_{2}\right\|
\end{aligned}
$$

by Theorem 1.3.20(ii). Thus $*$ is an algebraic $4 \varepsilon$-perturbation of $B$.

Theorem 1.4.5 Let $A$ be a real or complex Banach algebra with unit 1, and $*$ be an algebraic $\varepsilon$-perturbation of $A$. Suppose that one of the following conditions holds.

(i) $A$ is a complex function algebra on a compact Hausdorff space $X$, and $\varepsilon$ $<1$.

(ii) $A$ is a real function algebra on a compact Hausdorff space $X$ with a topological involution $\tau$, and $\varepsilon<1 / 4$.

(iii) $A$ is a real uniform algebra, there exists a positive constant $k$ such that $\|f\|^{2} \leq k\left\|f^{2}+g^{2}\right\|$ for all $f, g$ in $A$, and $\varepsilon<1 / 2 k$. 
Then $*$ is commutative, and $(A, *)$ is a uniform algebra under an equivalent norm.

Proof. In view of Theorem 1.4.3(ii), it is enough to show that $*$ is commutative in all the cases.

Let condition (i) hold and $N(f)$ denote the spectral radius of $f$ in $(A, *)$. By Theorem 1.4.3(i), for all $f$ in $A$,

$$
\begin{aligned}
\|f\|^{2} \leq \frac{N(f)^{2}}{(1-\varepsilon)^{2}} & =\frac{N(f * f)}{(1-\varepsilon)^{2}} \\
& =\frac{(1+\varepsilon)\|f * f\|}{(1-\varepsilon)^{2}} .
\end{aligned}
$$

Now by Theorem 1.1.30(i), * is commutative.

Next, let condition (ii) hold and $B:=\{f+i g: f, g \in A\}$. Then $B$ is a complex function algebra, and by Theorem 1.4 .4 , $*$ is an algebraic $4 \varepsilon-$ perturbation of $B$. Since $4 \varepsilon<1$, * is commutative by (i).

Finally, suppose that condition (iii) holds. Then for $f, g$ in $A$,

$$
\begin{aligned}
\|f\|^{2} & \leq k\left\|f^{2}+g^{2}\right\| \\
& \leq k\left(\left\|f^{2}-f * f\right\|+\|f * f+g * g\|+\left\|g * g-g^{2}\right\|\right) \\
& \leq k\|f * f+g * g\|+\varepsilon k\left(\|f\|^{2}+\|g\|^{2}\right)
\end{aligned}
$$

and similarly for $\|g\|^{2}$. Hence

$$
\|f\|^{2} \leq\|f\|^{2}+\|g\|^{2} \leq \beta\|f * f+g * g\|,
$$

where $\beta=2 k /(1-2 \varepsilon k)>0$ as $2 k \varepsilon<1$. Hence $*$ is commutative by Theorem 1.1.30(ii).

The proof of part (ii) of Theorem 1.4.5 is an illustration of employing the complexification technique to study the effect of perturbation on a property (in this case, commutativity) of a real function algebra. Some more examples of this technique can be found in Kulkarni and Arundhathi (1991-c).

We do not know whether the conclusion (ii) of Theorem 1.4.5 holds if $1 / 4 \leq \varepsilon<1$.

Note that if $X$ is a compact Hausdorff space, then $\|f\|^{2} \leq\left\|f^{2}+g^{2}\right\|$ for all $f, g$ in $C_{\beta}(X)$. Hence by part (iii) of Theorem 1.4.5, every algebraic $\varepsilon$-deformation of $C_{\text {用 }}(X)$ with $\varepsilon<1 / 2$ is commutative.

Theorems 1.4.3 and 1.4.5 raise the following natural questions about metric perturbations and commutativity.

1. Is every small metric perturbation of a function algebra $A$ a small algebraic perturbation? Precisely, is every metric $\varepsilon$-perturbation of $A$ 
with $0 \leq \varepsilon<\varepsilon_{0}$ for some fixed $\varepsilon_{0}$, an algebraic $k(\varepsilon)$-perturbation of $A$, where $k(\varepsilon)$ is a function of $\varepsilon$ such that $k(\varepsilon) \rightarrow 0$ as $\varepsilon \rightarrow 0$ ?

2. Is every small metric perturbation of a real or complex function algebra commutative? (Precise formulation as above can be given.)

3. Is every small metric deformation of a real or complex function algebra also a real or complex function algebra?

Notice that if question 1 has the affirmative answer, then so do questions 2 and 3, in view of Theorems 1.4.3 and 1.4.5. Jarosz (1985) has shown that this, in fact, is the case for complex function algebras. Now we show that the situation is rather different for real function algebras.

Example 1.4.6 Let $A=\left(\Omega^{2},\|\cdot\|_{\infty}\right)$ with the coordinatewise multiplication. For a fixed $t$ with $0 \leq t \leq \frac{1}{2}$, define $\rho_{t}: \Omega^{2} \times \Omega^{2} \rightarrow \Omega^{2}$ by

$$
\begin{array}{r}
\rho_{t}\left(\left(x_{1}, y_{1}\right),\left(x_{2}, y_{2}\right)\right)=\left(x_{1} x_{2}-t\left(x_{1}-y_{1}\right)\left(x_{2}-y_{2}\right),\right. \\
\left.y_{1} y_{2}-t\left(x_{1}-y_{1}\right)\left(x_{2}-y_{2}\right)\right) .
\end{array}
$$

A direct computation shows that $A_{t}:=\left(\mathbb{R}^{2}, \rho_{t},\|\cdot\|_{\infty}\right)$ is a commutative Banach algebra with unit $(1,1)$. Let $T: A \rightarrow A_{t}$ be the identity map. Since $\|T\|\left\|T^{-1}\right\|=1<1+\varepsilon$ for every $\varepsilon>0, \rho_{t}$ is a metric $\varepsilon$-perturbation of $A$ for every $\varepsilon>0$. If the answer to question 1 were affirmative, there would exist positive constants $\varepsilon_{0}$ and a function $k(\varepsilon)$ defined for $0 \leq \varepsilon<$ $\varepsilon_{0}$ such that $k(\varepsilon) \rightarrow 0$ as $\varepsilon \rightarrow 0$ and

$$
\left\|\rho_{t}\left(\left(x_{1}, y_{1}\right),\left(x_{2}, y_{2}\right)\right)-\left(x_{1}, y_{1}\right)\left(x_{2}, y_{2}\right)\right\|_{\infty} \leq k(\varepsilon)\left\|\left(x_{1}, y_{1}\right)\right\|_{\infty}\left\|\left(x_{2}, y_{2}\right)\right\|_{\infty}
$$

for all $\left(x_{1}, y_{1}\right),\left(x_{2}, y_{2}\right) \in R^{2}$ and every $\varepsilon$ with $0 \leq \varepsilon<\varepsilon_{0}$. But this would mean that $\rho_{t}$ coincides with the original multiplication, which is false. Also,

$$
\rho_{t}((1,-1),(1,-1))=(1-4 t, 1-4 t)=0 \quad \text { if } t=\frac{1}{4} .
$$

Thus $A_{1 / 4}$, which is a metric $\varepsilon$-deformation of $A$ for every $\varepsilon>0$, is not a uniform algebra. This shows that the answers to questions 1 and 3 are negative for real function algebras.

The next example is related to question 2 .

Example 1.4.7 Let $X=\left\{x_{1}, x_{2}, x_{3}, x_{4}\right\}$ be equipped with the discrete topology. Then $X$ is a compact Hausdorff space. Define $\tau: X \rightarrow X$ by $\tau\left(x_{1}\right)$ $=x_{2}, \tau\left(x_{2}\right)=x_{1}, \tau\left(x_{3}\right)=x_{4}$, and $\tau\left(x_{4}\right)=x_{3}$. Then $\tau$ is an involution on $X$. Denote by $H$ the Banach algebra of real quaternions (see Example 1.1.4). Let $a=a_{1}+i a_{2}, b=b_{1}+i b_{2}$, and $f_{a, b}$ in $C(X, \tau)$ be defined by $f_{a, b}\left(x_{1}\right)=$ $a, f_{a, b}\left(x_{2}\right)=\bar{a}, f_{a, b}\left(x_{3}\right)=b$, and $f_{a, b}\left(x_{4}\right)=\bar{b}$. Then define $T: C(X, \tau) \rightarrow$ 
by $T\left(f_{a, b}\right)=(1+j)^{-1}\left(a_{1}+i a_{2}+j b_{1}+k b_{2}\right)$. Note that the constant function $f_{1,1}$ is the unit in $C(X, \tau)$, and $T\left(f_{1,1}\right)=f_{1,1}$.

Suppose that $\left\|f_{a, b}\right\| \leq 1$. Then $|a| \leq 1,|b| \leq 1$ and

$$
\begin{aligned}
\left\|T\left(f_{a, b}\right)\right\| & =\left\|(1+j)^{-1}\right\|\left\|a_{1}+i a_{2}+j b_{1}+k b_{2}\right\| \\
& =\frac{\sqrt{a_{1}^{2}+a_{2}^{2}+b_{1}^{2}+b_{2}^{2}}}{\sqrt{2}} \leq 1 .
\end{aligned}
$$

Hence $\|T\| \leq 1$. Since $T\left(f_{1,1}\right)=f_{1,1}$, we see that $\|T\|=1$. Also, for every $f_{a, b}$ in $C(X, \tau)$, we have

$$
\begin{aligned}
\left\|f_{a, b}\right\|^{2}=\max \left\{a_{1}^{2}+a_{2}^{2}, b_{1}^{2}+b_{2}^{2}\right\} & \leq a_{1}^{2}+a_{2}^{2}+b_{1}^{2}+b_{2}^{2} \\
& =2\left\|T\left(f_{a, b}\right)\right\|^{2} .
\end{aligned}
$$

This shows that $\left\|T^{-1}\right\| \leq \sqrt{2}$. Since $T\left(f_{1,0}\right)=(1+j)^{-1}$, we have $\left\|T^{-1}\right\|$ $=\sqrt{2}$. Thus $T$ induces a metric $\varepsilon$-perturbation on $C(X, \tau)$, where $\varepsilon=$ $\sqrt{2}-1$, given by

$$
f_{a, b} * f_{c, d}=T^{-1}\left(T\left(f_{a, b}\right) T\left(f_{c, d}\right)\right) \quad \text { for } f_{a, b}, f_{c, d} \text { in } C(X, \tau) \text {. }
$$

It is easy to see that $*$ is noncommutative. For example, let $g=f_{i,-i}$ and $h$ $=f_{-1, i}$. Then $T g=i, T h=j$. Since $(T g)(T h) \neq(T h)(T g)$, we see that $g *$ $h \neq h * g$.

Note that Example 1.4.7 does not settle question 2; that is, we do not know whether there exists $\varepsilon_{0}>0$ such that every metric $\varepsilon$-perturbation with $\varepsilon \leq \varepsilon_{0}$ is commutative. We only know that if such an $\varepsilon_{0}$ exists, it must be less than $\sqrt{2}-1$. 


\section{References}

1. N. L. Alling (1970), Real Banach algebras and nonorientable Klein surfaces-I, J. Reine Angew. Math. 241, 200-208. MR 41, 5972.

2. N. L. Alling and L. A. Campbell (1972), Real Banach algebras-II, Math. Z. $125,79-100$. MR 45, 4150 .

3. N. L. Alling and N. Greenleaf (1971), Foundations of the Theory of Klein Surfaces, Springer-Verlag, Berlin. MR 48, 11488.

4. N. L. Alling and B. V. Limaye (1972), Ideal theory on non-orientable Klein surfaces, Ark. Mat. 10, 277-292. MR 53, 3892.

5. R. Arens and I. Kaplansky (1948), Topological representation of algebras, Trans. Amer. Math. Soc. 63, 457-481. MR 11, 317.

6. S. Arundhathi (1988), Studies in real function algebras, Ph.D. thesis, Indian Institute of Technology, Madras, India.

7. S. Arundhathi (1990), A note on the peak points for real function algebras, Indian J. Pure Appl. Math. 21, 155-162. MR 91f, 46071.

8. S. Arundhathi and S. H. Kulkarni (1986), Analytic and harmonic maps into a topological space, Proc. Indian Acad. Sci. (Math. Sci.) 95, 37-40. MR 88h, 46096.

9. H. S. Bear (1965), A geometric characterization of Gleason parts, Proc. Amer. Math. Soc. 16, 407-412. MR 31, 5136.

10. H. S. Bear (1970), Lectures on Gleason parts, Lecture Notes in Mathematics 121, Springer-Verlag, Berlin. MR 41, 5950.

11. E. Behrends (1979), $M$-Structure and the Banach-Stone theorem, Lecture Notes in Mathematics 736, Springer-Verlag, Berlin. MR 81b, 46002. 
12. A. Bernard (1968), Une characterization de $C(X)$ paemi les algebres de Banach, C. R. Acad. Sci. Paris 267, 634-635. MR 38, 2601.

13. A. Bernard (1970), Fonctions qui opérent sur Re A, C. R. Acad. Sci. Paris 271, 1120-1122. MR 42, 5048.

14. A. Bernard (1972), Espace des parties réelles des éléments d'une algèbre de Banach de fonctions, J. Funct. Anal. 10, 387-409. MR 49, 7781.

15. E. Bishop (1961), A generalization of the Stone-Weierstrass theorem, Pacific J. Math. 11, 777-783. MR 24, A 3502.

16. F. F. Bonsall and J. Duncan (1973), Complete Normed Algebras, Springer-Verlag, Berlin. MR 54, 11013.

17. L. de Branges (1959), The Stone-Weierstrass theorem, Proc. Amer. Math. Soc. 10, 822-824. MR 22, 3970.

18. B. Brosowski and F. Deutsch (1981), An elementary proof of the StoneWeierstrass theorem, Proc. Amer. Math. Soc. 81, 89-92. MR 81j, 46086.

19. A. Browder (1969), Introduction to Function Algebras, W. A. Benjamin, New York. MR 39, 7431.

20. R. B. Burckel (1972), Characterizations of $C(X)$ among its subalgebras, Lecture Notes in Pure and Applied Mathematics, Vol. 6, Marcel Dekker, New York. MR 56, 1068.

21. R. B. Burckel (1984), Bishop's Stone-Weierstrass theorem, Amer. Math. Monthly. 91, 22-32. MR 85i, 46071.

22. J. Cerych (1986), A remark on linear codimensions of function algebras, Czechoslovak Math. J. 36, 511-515. MR 87j, 46097.

23. T. W. Gamelin (1969), Uniform Algebras, Prentice Hall, Englewood Cliffs, N.J. MR 53, 14137.

24. J. Garnett (1967), A topological characterization of Gleason parts, Pacific J. Math. 20, 59-63. MR 34, 4942.

25. I. M. Gelfand (1941). Normierte ringe, Mat. Sbornik N.S. 9(51), 3-24. MR 3, 4486.

26. A. M. Gleason (1957), Function algebras, Seminars on Analytic Functions, Institute for Advanced Study, Princeton, N.J.

27. I. Glicksberg (1962), Measures orthogonal to algebras and sets of antisymmetry, Trans. Amer. Math. Soc. 105, 415-435. MR 30, 4164.

28. K. R. Goodearl (1982), Notes on Real and Complex $C^{*}$-Algebras, Shiva Publishing, Nantwich, Cheshire, England. MR 85d, 46079.

29. E. A. Gorin (1965), Moduli of invertible elements in a normed algebra, Vestnik Moskov. Univ. Ser. I Mat. Meh. 5, 35-39. MR 32, 8206.

30. M. Grzesiak (1986), Extreme points of the unit ball in the dual space of some real Banach algebra of continuous complex functions, Fasc. Math. 16, 5-10. MR 88c, 46062.

31. M. Grzesiak (1989), Real function algebras and their sets of antisymmetry, Glasnik Mat. 24, 297-304. MR 91i, 46055.

32. M. Grzesiak (1990), Isometries of a space of continuous functions determined by an involution, Math. Nachr. 145, 217-221. 
33. M. Grzesiak (1991), Localising families for real function algebras (to appear).

34. O. Hatori (1981), Functions which operate on the real part of a function algebra, Proc. Amer. Math. Soc. 83, 565-568. MR 82j, 46063.

35. R. A. Hirschfeld and W. Zelazko (1968), On spectral norm Banach algebras, Bull. Acad. Polon. Sci. 16, 195-199. MR 37, 4621.

36. K. Hoffman and J. Wermer (1962), A characterization of $C(X)$, Pacific J. Math. 12, 941-944. MR 27, 325.

37. S. Hwang (1990), Aspects of commutative Banach algebras, Ph.D. thesis, University of Connecticut.

38. L. Ingelstam (1962), A vertex property for Banach algebras with identity, Math. Scand. 2, 22-32. MR 26, 4199.

39. L. Ingelstam (1963), Hilbert algebras with identity, Bull. Amer. Math. Soc. 69, 794-796. MR 27, 4096.

40. L. Ingelstam (1964), Real Banach algebras, Ark. Mat. 5, 239-270. MR 30, 2358.

41. L. Ingelstam (1968), Symmetry in real Banach algebras, Math. Scand. 18, 53-68. MR 34, 6555.

42. L. Ingelstam (1967), A note on Laplace transforms and strict reality in Banach algebras, Math. Z. 102, 163-165. MR 36, 6936.

43. L. Ingelstam (1969). On semigroups generated by topological nilpotent elements, Illinois J. Math. 13, 172-175. MR 38, 4996.

44. K. Jarosz (1983), The uniqueness of multiplication in function algebras, Proc. Amer. Math. Soc. 89, 249-253. MR 85c, 46048.

45. K. Jarosz (1984a), A characterization of weak peak sets for function algebras, Bull. Austral. Math. Soc. 29, 129-135. MR 86a, 46058.

46. K. Jarosz (1984b), Into isomorphisms of spaces of continuous functions, Proc. Amer. Math. Soc. 90, 373-377. MR 85k, 46024.

47. K. Jarosz (1985), Perturbations of Banach algebras, Lecture Notes in Mathematics 1120, Springer-Verlag, Berlin. MR 86k, 46074.

48. K. JarosZ and Z. Sawon (1985), A discontinuous function does not operate on the real part of a function algebra, Casopis Pest. Mat. Soc. 110, 58-59. MR 86g, 46080.

49. B. E. Johnson (1977), Perturbations of Banach algebras, Proc. London Math. Soc. 34, 439-458. MR 56, 1094.

50. R. V. Kadison (1951), A representation theory for a commutative topological algebra, Mem. Amer. Math. Soc. 7, 39-50. MR 13, 360.

51. R. V. Kadison and D. Kastler (1972), Perturbations of von Newmann algebra I-stability of type, Amer. J. Math. 94, 38-54. MR 45, 5772.

52. I. Kaplansky (1949), Normed algebras, Duke Math. J. 16, 399-418. MR $11,115$.

53. H. Konig (1966), Zur abstracten Theorie analytischen Funktionen II, Math. Ann. 163, 9-17. MR 32, 8202.

54. M. König (1969), On the Gleason and Harnak metrics for uniform algebras, Proc. Amer. Math. Soc. 22, 100-101. MR 39, 3313. 
55. S. H. Kulkarni (1983), Analytic and harmonic maps into the maximal ideal space of a function algebra, J. Math. Phys. Sci. 17, 169-175. MR $84 j, 46081$.

56. S. H. Kulkarni (1988), Topological conditions for commutativity of a real Banach algebra, Houston J. Math. 14, 235-245. MR 90b, 46069.

57. S. H. Kulkarni and S. Arundhathi (1988), Choquet boundary for real function algebras, Canad. J. Math. 40, 1084-1104. MR 90a, 46129.

58. S. H. Kulkarni and S. Arundhathi (1991a), Isometries of real function algebras, Comment. Math. 30, 343-356.

59. S. H. Kulkarni and S. Arundhathi (1991b), A note on the metric topology on Gleason parts of a real function algebra (to appear).

60. S. H. Kulkarni and S. Arundhathi (1991c), Perturbations of real function algebras (to appear).

61. S. H. Kulkarni and B. V. Limaye (1980), Gelfand-Naimark theorems for real Banach algebras, Math. Japon. 25, 545-558. MR 82c, 46071.

62. S. H. Kulkarni and B. V. Limaye (1981a), Spectral mapping theorem for real Banach algebras, Houston J. Math. 7, 507-517. MR 83i, 46057.

63. S. H. Kulkarni and B. V. Limaye (1981b), Gleason parts of real function algebras, Canad. J. Math. 33, 181-200. MR 82m, 46052.

64. S. H. Kulkarni and B. V. Limaye (1983), A topological characterization of Gleason parts of real function algebras, Canad. Math. Bull. 26, 44-49. MR $83 m, 46076$.

65. S. H. Kulkarni and B. V. Limaye (1991a), Choquet sets and boundaries for real subspaces of $C(X)$ (to appear).

66. S. H. Kulkarni and B. V. Limaye (1991b), Extreme points of the unit ball of the dual space of a subspace of $C(X)$ (to appear).

67. S. H. Kulkarni and N. Srinivasan (1987), An analogue of Bishop's theorem for real function algebras, Indian J. Pure Appl. Math. 18, 136-145. MR 83d, 46101.

68. S. H. Kulkarni and N. Srinivasan (1988a), An analogue of HoffmanWermer theorem for real function algebras, Indian J. Pure Appl. Math. 19, 154-166. MR 89i, 46059.

69. S. H. Kulkarni and N. Srinivasan (1988b), A note on a theorem of Gorin, Math. Japon. 33, 887-893. MR 90a, 46130.

70. S. H. Kulkarni and N. Srinivasan (1990), An analogue of Wermer's theorem for a real function algebra, Math. Today 8, 33-42.

71. K. de Leeuw and Y. Katznelson (1963), Functions that operate on nonselfadjoint algebras, J. Analyse Math. 11, 207-219. MR 28, 1508.

72. B. V. Limaye (1976), Boundaries for real Banach algebras, Canad. J. Math. 28, 42-49. MR 52, 15022.

73. B. V. Limaye (1981), Functional Analysis, Wiley Eastern, New Delhi. MR $83 b, 46001$.

74. B. V. Limaye, R. D. Mehta, and M. H. Vasavada (1982), Maximal ideal space and Silov boundary of the tensor product of real Banach algebras, Glas. Mat. 17, 277-283. MR 84h, 46069. 
75. B. V. Limaye and R. R. Simha (1975), Deficiencies of certain real uniform algebras, Canad. J. Math. 27, 121-132. MR 52, 3962.

76. S. Machado (1977), On Bishop's generalization of the Stone-Weierstrass theorem, Nederl. Akad. Wetensch. Proc. Ser. A 80, Indag. Math. 39, 218-224. MR 56, 6356.

77. S. Mazur and S. Ulam (1932), Sur les transformations isométriques d'espace vectoriels normes, C. R. Acad. Sci. Paris, 194, 946-948.

78. M. S. Mehta, R. D. Mehta, and M. H. Vasavada (1990), Silov and other decompositions for a real function algebra, Math. Today 8, 1-12.

79. R. D. Mehta and M. H. Vasavada (1986), Wermer's type result for a real Banach function algebra, Math. Today 4, 43-46. MR 86c, 46064.

80. M. Nagasawa (1959), Isomorphisms between commutative Banach algebras with an application to rings of analytic functions, Kodai Math. Sem. Rep. 11, 182-188. MR 22, 12379.

81. H. W. Oliver (1970), Noncomplex methods in real Banach algebras, J. Funct. Anal. 6, 401-411. MR 42, 3581.

82. T. Ono (1970), A real analogue of the Gelfand-Naimark theorem, Proc. Amer. Math. Soc. 25, 159-160. MR 41, 2407.

83. C. Le Page (1967), Sur quelques conditions entraînant la commutativite dans les algèbres des Banach, C. R. Acad. Sci. Paris Ser. A-B 265, 235237. MR 37, 1999.

84. T. W. Palmer (1972), Real C* algebras, Pacific J. Math. 6, 245-290.

85. R. R. Phelps (1966), Lectures on Choquet's theorem. D. Van Nostrand, Princeton, N.J. MR 33, 1690.

86. J. Phillips (1973), Perturbations of $C^{*}$ algebras, Indiana Univ. Math. J. 23, 1167-1176. MR 49, 5861.

87. J. B. Prolla (1971), Bishop's generalized Stone-Weierstrass theorem for weighted spaces, Math. Ann. 191, 283-289. MR 44, 7200.

88. J. B. Prolla (1988), A generalized Bernstein approximation theorem, Math. Proc. Cambridge Philos. Soc. 104, 317-330. MR 90b, 41055.

89. I. Raeburn and J. L. Taylor (1977), Hochschild cohomology and perturbations of Banach algebras, J. Funct. Anal. 25, 258-267. MR 58, 30334.

90. T. J. Ransford (1984), A short elementary proof of the Bishop-StoneWeierstrass theorem, Math. Proc. Cambridge Philos. Soc. 96, 309-311. MR 86c, 46023.

91. C. E. Rickart (1960), General Theory of Banach Algebras, D. Van Nostrand, Princeton, N.J., MR 22, 5903.

92. R. Rochberg (1979), Deformation of uniform algebras, Proc. London Math. Soc. 39, 93-118. MR 80k, 46058.

93. W. Rudin (1957), The closed ideals in an algebra of analytic functions, Canad. J. Math. 9, 426-434. MR 19, 641.

94. W. Rudin (1964), Principles of Mathematical Analysis, McGraw-Hill, New York. MR 29, 3587.

95. W. Rudin (1966), Real and Complex Analysis, McGraw-Hill, New York. MR 35, 1420. 
96. W. Rudin (1973), Functional Analysis, McGraw-Hill, New York. MR S1, 1315.

97. Z. Semadeni (1971), Banach Spaces of Continuous Functions, PWNPolish Scientific Publishers, Warsaw. MR 45, 5930.

98. G. E. Shilov (1951), On rings of functions with uniform convergence, Ukrain. Mat. Z. 3, 404-411. MR 14, 884.

99. S. J. Sidney (1979), Functions which operate on a real part of a uniform algebra, Pacific J. Math. 80, 265-272. MR 81b, 46069.

100. S. J. Sidney and E. L. Stout (1968), A note on interpolation, Proc. Amer. Math. Soc. 19, 380-382. MR 81b, 46069.

101. G. F. Simmons (1963), Introduction to Topology and Modern Analysis, McGraw-Hill, New York. MR 26, 4145.

102. G. Springer (1957), Introduction to Riemann Surfaces, Addison-Wesley, Reading, Massachusetts. MR 19, 1169.

103. N. Srinivasan (1988), Characterizations of $C(X, \tau)$ among its subalgebras, $\mathrm{Ph} . \mathrm{D}$. thesis, Indian Institute of Technology, Madras, India.

104. N. Srinivasan and S. H. Kulkarni (1988), Restriction algebras of a real function algebra, J. Math. Phys. Sci. 22, 209-223. MR 89k, 46087.

105. A. Srivastav (1990), Commutativity criterion for real Banach algebras, Arch. Math. 54, 65-72. MR 91b, 46044.

106. A. Srivastav (1991), Extreme points of positive functionals and spectral states on real Banach algebras (to appear).

107. E. L. Stout (1971), The Theory of Uniform Algebras, Bogden and Quigley, Tarrytown-on-Hudson, N.Y. MR 54, 11066.

108. I. Suciu (1975), Function Algebras, Noordhoff International Publishing, Leyden, The Netherlands. MR 51, 6248.

109. J. Wermer (1963), The space of real parts of a function algebra, Pacific $J$. Math. 13, 1423-1426. MR 27, 6152.

110. W. Zelazko (1973), Banach algebras PWN-Polish Scientific Publishers, Warsaw. MR 56, 6389. 